\title{
Perturbations of Flows on Banach Spaces and Operator Algebras $\star \star \star$
}

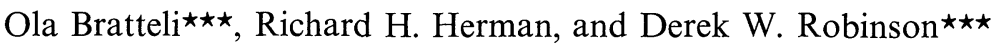 \\ Department of Mathematics, Pennsylvania State University, \\ University Park, Pennsylvania 16802, USA
}

\begin{abstract}
For automorphism groups of operator algebras we show how properties of the difference $\left\|\alpha_{t}-\alpha_{t}^{\prime}\right\|$ are reflected in relations between the generators $\delta_{\alpha}, \delta_{\alpha}^{\prime}$. Indeed for a von Neumann algebra $\mathscr{M}$, with separable predual we show that if $\left\|\alpha_{t}-\alpha_{t}^{\prime}\right\| \leqq 0.28$ for small $t$, then $\delta_{\alpha}=\gamma \circ\left(\delta_{\alpha}^{\prime}+\delta\right) \circ \gamma^{-1}$ where $\gamma$ is an inner automorphism of $\mathscr{M}$ and $\delta$ is a bounded derivation of $\mathscr{M}$. If the difference $\left\|\alpha_{t}-\alpha_{t}^{\prime}\right\|=O(t)$ as $t \rightarrow 0$, then $\delta_{\alpha}=\delta_{\alpha}^{\prime}+\delta$ and if $\left\|\alpha_{t}-\alpha_{t}^{\prime}\right\| \leqq 0.28$ for all $t$ then $\delta_{\alpha}=\gamma \circ \delta_{\alpha}^{\prime} \circ \gamma^{-1}$. We prove analogous results for unitary groups on a Hilbert space and $C_{0}, C_{0}^{*}$ groups on a Banach space.
\end{abstract}

\section{§1. Introduction}

Questions of perturbations of dynamics have received considerable attention from various points of view. In this paper we will consider one parameter groups of operators acting on dual Banach spaces and one parameter groups of *-automorphism of operator algebras. Recent considerations have centered on studying the behaviour of the difference of two dynamics as the parameter $t$ goes to zero. Bucholz and Roberts [5] consider the situation where $\left\|\alpha_{t}-\alpha_{t}^{\prime}\right\| \rightarrow 0$ as $t \rightarrow 0$, where $\alpha_{t}, \alpha_{t}^{\prime}$ are *-automorphism groups of a simple $C^{*}$-algebra or a von Neumann algebra. Among other things they show that the generator of $\alpha_{t}$ is related to that of $\alpha_{t}^{\prime}$ by twisting and then adding a bounded perturbation. In [14] Robinson considers the question of the proximity of $C_{0}$ semi-groups of operators, $U_{t}, V_{t}$ on a Banach space and characterizes $\left\|U_{t}-V_{t}\right\|=O\left(t^{\alpha}\right), 0<\alpha \leqq 1$. One also sees in [14] that the pointwise behaviour of $U_{t}-V_{t}$, i.e. the behaviour of $\left\|\left(U_{t}-V_{t}\right)(x)\right\|$ for $x$ belonging to the domain of the generator of $V_{t}$ is worth studying. Other aspects of perturbation of dynamics has been studied in [20]; and in [21] (concluding remarks).

For automorphism groups of von Neumann algebras our results fall into three categories. We consider the cases where $\left\|\alpha_{t}-\alpha_{t}^{\prime}\right\|$ is, small for $t$ small, small for all $t$,

\footnotetext{
$\star \quad$ This paper subsumes an earlier work of the same title which appeared as a report from Z.I.F. der Universität Bielefeld

$\star \star \quad$ With partial support of the U.S. National Science Foundation

$\star \star \star$ Permanent address : Dept. de Physique, Univ. d'Aix-Marseille II, Luminy, F-Marseille, and Centre de Physique Théorique, CNRS, 31, Chemin J. Aiguier, F-13 Marseille, France
} 
or $O(t)$ as $t \rightarrow 0$. Results of Kadison and Ringrose suggest consideration of the first case. We deal with the first two for von Neumann algebras with separable pre-dual as we are in need of Borel cross-section theorems. The last two situations are in a sense part of the first. More precisely; if $\left\|\alpha_{t}-\alpha_{t}^{\prime}\right\|$ is small for $t$ small then (Theorem 3.6) $\delta_{\alpha}=\gamma \circ\left(\delta_{\alpha}^{\prime}+\delta\right) \circ \gamma^{-1}$ where $\delta_{\alpha}, \delta_{\alpha}^{\prime}$ are the generators of $\alpha_{t}, \alpha_{t}^{\prime}$ respectively, $\delta$ is a bounded derivation and $\gamma$ is an inner automorphism of the von Neumann algebra. Note that this is the same form as the main result of [5], where it is assumed that that the difference $\left\|\alpha_{t}-\alpha_{t}^{\prime}\right\|$ goes to zero or $t \rightarrow 0$. The difference in these two cases is precisely the continuity of the orbit of the unitary giving $\gamma$, under $\alpha_{t}$. The last two cases represent "parts" of the one just described. If the automorphisms are close (enough) for all $t$ then $\delta_{\alpha}=\gamma \circ \delta_{\alpha^{\prime}} \circ \gamma^{-1}$; (Theorem 3.5) if the difference $\left\|\alpha_{t}-\alpha_{t}^{\prime}\right\|$ is $O(t)$ as $t \rightarrow 0$ then $\delta_{\alpha}=\delta_{\alpha^{\prime}}+\delta$ (Theorem 3.1). Here $\gamma$ is again an inner automorphism and $\delta$ a bounded derivation. Theorem 3.5 generalizes a result of Reynolds [26].

For von Neumann algebras in standard form $O(t)$ behaviour yields an explicit relation for the generators of the canonical unitaries. Indeed this shows that Araki's [3] perturbation of the modular automorphism group is characterized by $O(t)$ behaviour of the difference. One might incline to the view that $O(t)$ behaviour for automorphism groups of simple $C^{*}$-algebras would yield the same result as in the von Neumann algebra case. This however fails totally, as examples show. In some cases, e.g. quasi-free automorphisms of the Clifford algebra (Theorem 4.3) the result is the same as for von Neumann algebras.

The general results for automorphism groups have analogs for unitary groups in Hilbert space (indeed we need these for the former). These results are discussed in Section 2.

Finally we deal with examples which show that the above situations arise. One of the examples shows that it is possible to have $\left\|\alpha_{t}-\alpha_{t}^{\prime}\right\|=a$ for all $t \in \boldsymbol{R} \backslash\{0\}$ where $0 \leqq a \leqq 2$.

\section{§2. Banach and Hilbert Space Theory}

In this section we deal with unitary groups and $C_{0}$ or $C_{0}^{*}$ groups. The results we obtain for unitary groups will be used in our treatment of automorphism groups of von Neumann algebras in Section 3.

Recall [6] that a $C_{0}$-semigroup, $U_{t}$, of operators on a Banach space $X$ is a homomorphism from $\boldsymbol{R}$ into the bounded operators on $X$ such that $t \rightarrow U_{t} x$ is continuous and $U_{0}=I$ (the identity operator). A $C_{0}^{*}$-group is a dual group of a $C_{0}$-group.

Theorem 2.1. Let $U, V$ be two $C_{0}$ or $C_{0}^{*}$ groups, operating on a Banach space $X$, with generators $S, T$ respectively and let I denote this identity operator.

The following conditions are equivalent:

1. There exists $\varepsilon_{1}, 0<\varepsilon_{1}<1$ and $\delta_{1}>0$ such that

$$
\left\|U_{t} V_{-t}-I\right\| \leqq 1-\varepsilon_{1}
$$

for $0 \leqq t \leqq \delta_{1}$.

2. There exists $\varepsilon_{2}, 0<\varepsilon_{2}<1, \delta_{2}>0$, a bounded operator $P: \mathscr{D}(T) \rightarrow X$ and a bounded operator $\Omega: X \rightarrow X$ with bounded inverse, such that

$$
S=\Omega(T+P) \Omega^{-1}
$$


and

$$
\left\|U_{t} \Omega^{-1} U_{-t} \Omega-I\right\| \leqq 1-\varepsilon_{2}, \quad 0 \leqq t \leqq \delta_{2} .
$$

Under these conditions $\Omega$ may be defined by $\Omega=\frac{1}{\delta_{1}} \int_{0}^{\delta_{1}} d s U_{s} V_{-s}$. One has

$$
\begin{aligned}
& \|I-\Omega\| \leqq 1-\varepsilon_{1}, \\
& \left\|U_{t} \Omega^{-1} U_{-t} \Omega-I\right\| \leqq\left\|U_{t} V_{-t}-I\right\|+O(t), \text { as } t \rightarrow 0 \\
& \left\|U_{t} \Omega V_{-t}-\Omega\right\|=O(t) \text { as } t \rightarrow 0 .
\end{aligned}
$$

Proof. $1 . \Rightarrow 2$.

Define

$$
\Omega=\frac{1}{\delta_{1}} \int_{0}^{\delta_{1}} d s U_{s} V_{-s}
$$

It follows that $\|I-\Omega\| \leqq 1-\varepsilon_{1}$ and so $\Omega$ is a bounded operator with bounded inverse. Introduce $\chi_{t}$ by

$$
\chi_{t}=\Omega^{-1} U_{t} \Omega V_{t}
$$

As in [14]

$$
\frac{\chi_{t+h}-\chi_{t}}{h}=\frac{1}{\delta_{1} h} \Omega^{-1} \int_{\delta_{1}}^{\delta_{1}+h} d s U_{s+t} V_{-s-t}-\frac{1}{\delta_{1} h} \Omega^{-1} \int_{0}^{h} d s U_{s+s} V_{-s-t} .
$$

This implies that $\chi_{t}$ is strongly differentiable in the $C_{0}$-case and weak*differentiable in the $C_{0}^{*}$-case. The derivative is given by

$$
\frac{d \chi_{t}}{d t}=\Omega^{-1} U_{t}\left(U_{\delta_{1}} V_{-\delta}-I\right) V_{-t} / \delta_{1}
$$

For $x \in X$ we have

$$
\frac{\left(U_{t}-I\right) \Omega x}{t}=\frac{\Omega\left(V_{t}-I\right) x}{t}+\frac{\Omega\left(\chi_{t}-I\right) V_{t} x}{t},
$$

so that if $x \in \mathscr{D}(T)$ the right hand side converges, showing that $\Omega x \in \mathscr{D}(S)$ and

$$
S \Omega x=\Omega T x+\Omega P x .
$$

Here

$$
P=\left.\frac{d \chi_{t}}{d t}\right|_{t=0}=\Omega^{-1}\left(U_{\delta_{1}} V_{-\delta_{1}}-I\right) / \delta_{1} .
$$

Similarly if $x \in \mathscr{D}(S)$ then $\Omega^{-1} x \in \mathscr{D}(T)$ and

$$
\Omega^{-1} S x=T \Omega^{-1} x+P \Omega^{-1} x .
$$

We thus conclude that

$$
\mathscr{D}(S)=\Omega \mathscr{D}(T) \quad \text { and } \quad S=\Omega(T+P) \Omega^{-1} .
$$


Finally one computes that

$$
\begin{aligned}
U_{t} \Omega^{-1} U_{-t} \Omega-I= & \left(U_{t} V_{-t}-I\right)\left(V_{t} \Omega^{-1} U_{-t} \Omega-I\right) \\
& +\frac{1}{\delta_{1}} \Omega^{-1}\left[\int_{0}^{t} d s U_{s}\left(I-U_{\delta_{1}} V_{-\delta_{1}}\right) V_{-s}\right] V_{t} \Omega^{-1} U_{-t} \Omega \\
& +\left(U_{t} V_{-t}-I\right) .
\end{aligned}
$$

The existence of $\varepsilon_{2}, \delta_{2}$ is now clear. If one notes that $\Omega^{-1} U_{t} \Omega$ is the group of operators with generator $T+P$, perturbation theory immediately yields that

(*) $\left\|V_{t} \Omega^{-1} U_{-t} \Omega-I\right\|=O(t), \quad t \rightarrow 0$

and thus

(**) $\left\|U_{t} \Omega^{-1} U_{-t} \Omega-I\right\| \leqq\left\|U_{t} V_{-t}-I\right\|+O(t)$,

yielding the final statements of the theorem.

$2 . \Rightarrow 1$.

Define $Q=-\Omega P \Omega^{-1}$. Thus $T=\Omega^{-1}(S+Q) \Omega$. If $\hat{U}$ is the group generated by $S+Q$,

$$
U_{t} V_{-t}-I=U_{t} \Omega^{-1} U_{-t}\left(U_{t} \hat{U}_{-t}-I\right) \Omega+\left(U_{t} \Omega^{-1} U_{-t} \Omega-I\right) \text {. }
$$

Perturbation theory gives $\left\|U_{t} \hat{U}_{-t}-I\right\|=O(t)$, as $t \rightarrow 0$ and therefore

$$
\left\|U_{t} V_{-t}-I\right\| \leqq\left\|U_{t} \Omega^{-1} U_{-t} \Omega-I\right\|+O(t), \quad t \rightarrow 0
$$

completing the proof.

For unitary groups on a Hilbert space Theorem 2.1 can be improved.

Theorem 2.2. Let $U_{t}=\exp i t H, V_{t}=\exp i t K$ be strongly continuous unitary groups on a Hilbert space.

The following conditions are equivalent:

1. There exists $\varepsilon_{1}, 0<\varepsilon_{1}<\sqrt{2}$ and $\delta_{1}>0$ such that

$$
\left\|U_{t}-V_{t}\right\| \leqq \sqrt{2}-\varepsilon, \quad 0 \leqq t \leqq \delta_{1} \text {. }
$$

2. There exists $\varepsilon_{2}, 0<\varepsilon_{2}<\sqrt{2}, \delta_{2}>0$, a bounded self-adjoint operator $P$ and $a$ unitary operator $W$ such that

$$
\begin{aligned}
& H=W(K+P) W^{*} \\
& \left\|U_{t} W^{*} U_{-t} W-I\right\| \leqq \sqrt{2}-\varepsilon_{2}, \quad 0 \leqq t \leqq \delta_{2} .
\end{aligned}
$$

If these conditions are satisfied, then $W$ can be chosen as the unitary operator occuring in the polar decomposition of the invertible operator

$$
\Omega=\frac{1}{\delta_{1}} \int_{0}^{\delta_{1}} d t U_{t}-V_{-t}
$$

Moreover $\|W-I\| \leqq \sqrt{2}-\varepsilon_{1}$.

Proof. The proof that $1 . \Rightarrow 2$. relies on the following lemma. 
Lemma 2.3 (U. Haggerup). Let $\mathscr{U}$ be a collection of unitaries on a Hilbert space $\mathscr{H}$ and assume there exists an $\varepsilon>0$ such that if $U \in \mathscr{U}$ then $\|U-I\|<\sqrt{2}-\varepsilon$. If $\Omega$ is an operator in the $\sigma$-weakly closed convex hull of $\mathscr{U}$ then $\Omega$ is invertible with bounded inverse.

Proof. For any bounded operator $A$ on $\mathscr{H}$, let $W(A)=\{(A \psi, \psi) ;\|\psi\|=1\}$ be its numerical range. Then for any $U \in \mathscr{U}, W(U)$ is contained in the convex set

$$
\left\{z \in C ;|z| \leqq 1, \operatorname{Re} z \geqq \sqrt{2} \varepsilon-\frac{\varepsilon^{2}}{2}\right\} .
$$

Thus $W(\Omega)$ is contained in this set. As $\operatorname{Sp}(\Omega) \cong W(\Omega)$, [32], it follows that $\Omega$ is invertible.

Now we return to the proof of the theorem. Consider the operator

$$
\Omega=\frac{1}{\delta_{1}} \int_{0}^{\delta_{1}} d s U_{s} V_{-s} .
$$

It follows from the assumptions on $\left\|U_{t} V_{-t}-I\right\|$, and the above lemma, that $\Omega$ is invertible. The calculations of Theorem 2.1 show that

$$
\left\|U_{t} \Omega V_{-t}-\Omega\right\|=O(t)=\left\|V_{t} \Omega^{*} U_{-t}-\Omega^{*}\right\| \text {. }
$$

Thus

$$
\begin{aligned}
\left\|V_{t} \Omega^{*} \Omega V_{-t}-\Omega^{*}\right\|= & \left\|\left(V_{t} \Omega^{*} U_{-t}\right)\left(U_{t} \Omega V_{t}\right)-\Omega^{*} \Omega\right\| \\
& \leqq\left\|V_{t} \Omega^{*} U_{-t}-\Omega^{*}\right\|\left\|U_{t} \Omega V_{-t}\right\| \\
& +\left\|U_{t} \Omega V_{-t}-\Omega\right\|\left\|\Omega^{*}\right\|=O(t) \text { as } t \rightarrow 0 .
\end{aligned}
$$

This implies [12] that $|\Omega|^{2}$ lies in the domain of the derivation generating the automorphism group of $\mathscr{L}(\mathscr{H})$, implemented by $V_{t}$. From [21], we see that $|\Omega|^{-1}=\left(|\Omega|^{2}\right)^{-1 / 2}$ lies in the domain of that derivation and hence

$$
\left\|V_{t}|\Omega|^{-1} V_{-t}-|\Omega|^{-1}\right\|=O(t), \quad \text { as } t \rightarrow 0 .
$$

Taking $W$ to be the unitary occuring in the polar decomposition of $\Omega$ we have

$$
\begin{aligned}
\left\|U_{t} W V_{-t}-W\right\|= & \left\|U_{t} \Omega V_{-t} V_{t}|\Omega|^{-1} V_{-t}-\Omega|\Omega|^{-1}\right\| \\
& \leqq\left\|U_{t} \Omega V_{-t}-\Omega\right\|\left\||\Omega|^{-1}\right\| \\
& +\|\Omega\|\left\|V_{t}|\Omega|^{-1} V_{-t}-|\Omega|^{-1}\right\| \\
= & O(t) .
\end{aligned}
$$

Introducing $\hat{V}_{t}=W V_{t} W^{*}$ and noting that $\left\|U_{t}-\hat{V}_{t}\right\|=O(t)$, we have that $([14$, Corollary 3]) the generators of $U_{t}$ and $\hat{V}_{t}$ differ by a bounded self-adjoint operator. This establishes the relation between $H$ and $K$. Further we see that

$$
\left\|U_{t} W^{*} U_{-t}-W^{*}\right\|=\left\|U_{t} W U_{-t}-W\right\|
$$

and

$$
\left\|U_{t} W U_{-t}-W\right\| \leqq\left\|\left(U_{t} W_{-t}-W\right) V_{t} U_{-t}\right\|+\left\|W\left(V_{t} U_{-t}-I\right)\right\| .
$$


So by the above calculation the first term is $O(t)$ as $t \rightarrow 0$ and the second is smaller than $\sqrt{2}-\varepsilon_{1}$ for $t$ small. Thus $\delta_{2}$, and $\varepsilon_{2}$, exist.

2. $\Rightarrow 1$. proceeds as in the proof of Theorem 2.1.

We may now obtain our estimate in $\|W-I\|$ by appealing to a result of Woronowicz [33]. As noted $\Omega$ has numerical range in

$$
\left\{z \in \mathbb{C},|z| \leqq 1, \operatorname{Re} z \geqq \sqrt{2} \varepsilon_{1}-\frac{\varepsilon_{1}}{2}\right\} \text {. }
$$

However Woronowicz's result shows that the unitary $W$ has its spectrum there. Hence $\|W-I\| \leqq \sqrt{2}-\varepsilon_{1}$.

The next result shows that the perturbation $P$ in condition 2 of Theorem 2.2 can be eliminated if $U_{t}$ and $V_{t}$ are sufficiently close for all $t$.

After this paper was completed we found the paper [34], where a slightly weaker version of the following theorem is proved by the same method.

Theorem 2.4. Let $U_{t}, V_{t}$ be strongly continuous one-parameter unitary groups on $a$ Hilbert space. Assume that $\left\|U_{t}-V_{t}\right\| \leqq k$ for all $t \in \boldsymbol{R}$, where $k<\sqrt{2}$. It follows that there exists a unitary $W$ in the von Neumann algebra generated by $\left\{U_{t} V_{-t}, t \in \boldsymbol{R}\right\}$ such that

$$
U_{t}=W V_{t} W^{*}
$$

for all $t \in \boldsymbol{R}$. Moreover $\|W-I\| \leqq k$.

Proof. Let $\mathscr{M}$ be the von Neumann algebra generated by $U$ and $V$. Let $m$ be an invariant mean on $\boldsymbol{R}$ and define $\Omega=m_{s}\left(U_{s} V_{-s}\right)$. Since $\mathscr{M}=\left(\mathscr{M}_{*}\right) *$ it follows that $\Omega$ is well defined and $\Omega \in \mathscr{M}$. But since

$$
\left(U_{s} V_{-s}\right) V_{t}=U_{t}\left(U_{s-t} V_{-(s-t)}\right)
$$

it follows that $\Omega V_{t}=U_{t} \Omega$. An application of Lemma 2.3 shows that $\Omega$ is invertible and one then easily sees that $W V_{t}=U_{t} W$, where $W$ is the unitary occuring in the polar decomposition of $\Omega$.

The estimate on $\|W-I\|$ is obtained as in Theorem 2.3.

In Section 4 we will examine various examples which satisfy the conditions of Theorem 2.4.

We conclude this section by deriving a result on relatively bounded perturbations of $C_{0}^{*}$ semigroups.

We need to recall some of the general theory and terminology of adjoint semigroups. We refer the reader to [6] for the details.

Let $t \rightarrow U_{t}$ be a strongly continuous $\left(C_{0}\right)$ semigroup of operators on a Banach space $X$. The adjoint semigroup is $t \rightarrow U_{t}^{*}$ acting on the dual Banach space $X^{*}$. In general one no longer has strong continuity of the adjoint semigroup, but trivially it is weak *-continuous i.e. $t \rightarrow U_{t}^{*}(f)(x)$ is continuous for fixed $f$ in $X^{*}$ and $x$ in $X$. The general theory tells us that if $S$ is the generator of $U$ then $S^{*}$ is the weak 
*-generator of $U^{*}$. It turns out that the set of elements, $f \in X^{*}$, for which $t \rightarrow U_{t}^{*} f$ is strongly continuous, is a strongly closed, invariant weak*-dense, subspace $X^{*}(U)$ of $X^{*}$. Further $\mathscr{D}\left(S^{*}\right) \subseteq X^{*}(U)$.

Let $U_{0, t}^{*}$ denote the restriction of $U_{t}^{*}$ to $X^{*}(U)$. We write $S_{0}^{*}$ for its generator.

Theorem 2.5. Let $U, V$ be strongly continuous semigroups on a Banach space $X$, with infinitesimal generators $S, T$ respectively.

The following six conditions are equivalent:

1. (and $1_{0}$ )

$$
\left\|\left(U_{t}^{*}-V_{t}^{*}\right) f\right\|=O(t), \quad t \rightarrow 0
$$

for all $f \in \mathscr{D}\left(T^{*}\right)$ (for all $f \in \mathscr{D}\left(T_{0}^{*}\right)$ ).

2. (and 20).

One has $\mathscr{D}\left(S^{*}\right) \supseteqq \mathscr{D}\left(T^{*}\right)$ (one has $\mathscr{D}\left(S^{*}\right) \supseteqq \mathscr{D}\left(T_{0}^{*}\right)$ ) and there exist constants $a, b \geqq 0$ such that

$$
\left\|\left(S^{*}-T^{*}\right) f\right\| \leqq a\|f\|+b\left\|T^{*} f\right\|
$$

for all $f \in \mathscr{D}\left(T^{*}\right)$ ( for all $f \in \mathscr{D}\left(T_{0}^{*}\right)$ ).

3. The estimate

$$
\left\|\left(U_{t}^{*}-V_{t}^{*}\right)\left(1+\varepsilon T^{*}\right)^{-1}\right\|=O(t)
$$

is valid for all $\varepsilon$ in an interval $0<\varepsilon<\delta$.

4. The estimate

$$
\left\|(1+\varepsilon T)^{-1}\left(U_{t}-V_{t}\right)\right\|=O(t)
$$

is valid for all $\varepsilon$ in an interval $0<\varepsilon<\delta$.

Proof. The proof is based on an extension of methods used in [14] and ultimately relies on de Leeuw's characterization [12] of generators of adjoint semi-groups; $\mathrm{Viz}$

$$
f \in \mathscr{D}\left(S^{*}\right) \quad \text { if and only if } \quad\left\|\left(U_{t}^{*}-1\right) f\right\|=O(t) \text { as } t \rightarrow 0 .
$$

We show first that

$1 . \Rightarrow 2$.

If $f \in \mathscr{D}\left(T^{*}\right)$ then one has

$$
\left\|\left(1-U_{t}^{*}\right) f\right\| \leqq\left\|\left(1-V_{t}^{*}\right) f\right\|+\left\|\left(V_{t}^{*}-U_{t}^{*}\right) f\right\|=O(t) .
$$

Therefore $f \in \mathscr{D}\left(S^{*}\right)$, by our preliminary remarks, and so $\mathscr{D}\left(S^{*}\right) \supseteqq \mathscr{D}\left(T^{*}\right)$.

We now make use of Hörmander's comparison theorem (Theorem II.6.2 of [18]) to obtain the estimate in 2 .

Specifically we let

$$
\begin{gathered}
X_{0}=X_{1}=X_{2}=X^{*} \\
T_{1}=T^{*} \quad T_{2}=S^{*} .
\end{gathered}
$$

The operators $T^{*}$ and $S^{*}$ are weak* closed, thus strongly closed, and so we may apply the quoted theorem to obtain constants $a^{\prime}, b^{\prime} \geqq 0$ such that

$$
\left\|S^{*} f\right\| \leqq a^{\prime}\|f\|+b^{\prime}\left\|T^{*} f\right\|
$$


for all $f \in \mathscr{D}\left(T^{*}\right)$ or

2. $\left\|\left(T^{*}-S^{*}\right) f\right\| \leqq a\|f\|+b\left\|T^{*} f\right\|$

with $a=a^{\prime}, b=1+b^{\prime}$.

Note that we have used a version of Hörmander's theorem where it is not assumed that $T_{1}$ and $T_{2}$ are densely defined. The proof of this version is the same as the original one.

$2 . \Rightarrow 3$.

If $f \in \mathscr{D}\left(T^{*}\right)$ then $V_{t}^{*} f \in \mathscr{D}\left(T^{*}\right) \subseteq \mathscr{D}\left(S^{*}\right)$ and

$$
\left(U_{t}^{*}-V_{t}^{*}\right) f=\int_{0}^{t} d s U_{s}^{*}\left(T^{*}-S^{*}\right) V_{t-s}^{*} f
$$

Therefore,

$$
\begin{aligned}
\left\|\left(U_{t}^{*}-V_{t}^{*}\right) f\right\| & \leqq \int_{0}^{t} d s\left\|U_{s}^{*}\right\|\left\|\left(T^{*}-S^{*}\right) V_{t-s}^{*} f\right\| \\
& \leqq \int_{0}^{t} d s\left\|U_{s}\right\|\left(a\left\|V_{t-s}^{*} f\right\|+b\left\|T^{*} V_{t-s}^{*} f\right\|\right. \\
& \leqq \int_{0}^{t} d s\left\|U_{s}\right\|\left\|V_{t-s}\right\|\left(a\|f\|+b\left\|T^{*} f\right\|\right) .
\end{aligned}
$$

We know there exist constants $M$, $\omega$ such that

$$
\left\|U_{t}\right\| \leqq M e^{\omega t} ;\left\|V_{t}\right\| \leqq M e^{\omega t}
$$

and so we conclude that

$$
\left\|\left(U_{t}^{*}-V_{t}^{*}\right) f\right\| \leqq t M^{2} e^{\omega t}\left(a\|f\|+b\left\|T^{*} f\right\|\right) .
$$

Now if $1>\omega \varepsilon \geqq 0$ then

$$
\left\|(1+\varepsilon T)^{-1}\right\|=\left\|\int_{0}^{\infty} d t e^{-t} V_{-\varepsilon t}\right\| \leqq M(1-\omega \varepsilon)^{-1}
$$

and $\left\|T(1+\varepsilon T)^{-1}\right\| \leqq \varepsilon^{-1}\left(1+M(1-\omega \varepsilon)^{-1}\right)$.

If $g \in X^{*}$, we thus have the estimate,

$$
\begin{aligned}
& \left\|\left(U_{t}^{*}-V_{t}^{*}\right)\left(1+\varepsilon T^{*}\right)^{-1} g\right\| \\
& \quad \leqq t M^{2} e^{\omega t}\left\{a M(1-\omega \varepsilon)^{-1}+b \varepsilon^{-1}\left(1+M(1-\omega \varepsilon)^{-1}\right)\right\}\|g\| .
\end{aligned}
$$

Taking the supremum over $g$ of norm 1 we obtain 3 .

3. $\Leftrightarrow 4$. Clear.

3. $\Rightarrow 1$. This follows immediately from the fact that

$$
\mathscr{D}\left(T^{*}\right)=\mathscr{R}\left(\left(1+\varepsilon T^{*}\right)^{-1}\right) .
$$

$1_{0} \Leftrightarrow 2_{0} \Leftrightarrow 3$. 
If we begin with $1_{0}$. and repeat the reasoning of $1 . \Rightarrow 2$. we obtain $1_{0} . \Rightarrow 2$. To apply Hörmander's theorem one need only verify that $T_{0}^{*}$ is a closed operator. This is true since $T_{0}^{*}$ is the generator of $V_{0, t^{*}}^{*}$

A slight variation of the argument of $2 . \Rightarrow 3$. (we take the Laplace transform directly on the adjoint semi-group) yields

$$
\left\|\left(U_{t}^{*}-V_{t}^{*}\right)\left(1+\varepsilon T_{0}^{*}\right)^{-1}\right\|=O(t) .
$$

Now let $f \in X^{*}(V)$ and $A \in X$. Then

$$
\left(f, V_{t} A\right)=\left(V_{0, t}^{*} f, A\right) \text {. }
$$

Taking Laplace transforms, one finds that

$$
\begin{aligned}
\left(f,(1+\varepsilon T)^{-1} A\right) & =\int_{0}^{\infty} d t e^{-t}\left(f, V_{\varepsilon t} A\right) \\
& =\int_{0}^{t} d t e^{-t}\left(V_{0, t}^{*} f, A\right) \\
& =\left(\left(1+\varepsilon T_{0}^{*}\right)^{-1} f, A\right) .
\end{aligned}
$$

Now $X^{*}(V) \geqq \mathscr{D}\left(T^{*}\right)$, [6], and so $X^{*}(V)$ contains all elements of the form

$$
\int_{-\infty}^{\infty} d t E(t, s) V_{t}^{*} f
$$

where $E(t, s)=(2 \sqrt{\pi s})^{-1} \exp \left(-\frac{t^{2}}{4 s}\right)$. Since we know $\left\|V_{t}^{*}\right\| \leqq M e^{\omega t}$, we can renormalize the $E(t, s)$, to conclude that the unit ball of $X^{*}(V)$ is weak* dense in the unit ball of $X^{*}$, we can then conclude from the above equality that

$$
\left\|(1+\varepsilon T)^{-1}\right\|=\left\|\left(1+\varepsilon T_{0}^{*}\right)^{-1}\right\| .
$$

Essentially the same argument yields

$$
\begin{aligned}
\left\|(1+\varepsilon T)^{-1}\left(U_{t}-V_{t}\right)\right\| & =\left\|\left(U_{t}^{*}-V_{t}^{*}\right)\left(1+\varepsilon T_{0}^{*}\right)^{-1}\right\| \\
& =O(t)
\end{aligned}
$$

and then $1_{0} \Rightarrow 4$. However $4 . \Leftrightarrow 3$., then establishing $1 . \Rightarrow 3$.

Clearly $3 . \Rightarrow 1 . \Rightarrow 1_{0}$ and so the proof of the theorem is complete.

The reader is referred to [14] for an estimate on the relative bound of $S^{*}-T^{*}$.

\section{§3. Von Neumann Algebra Theory}

In this section we obtain the principal results cited in the introduction.

We first digress to define a constant $C(\alpha, \beta) \in[0, \infty]$, for any two $\sigma$-weakly continuous, one-parameter *automorphism groups, $\alpha_{t}, \beta_{t}$ of a von Neumann algebra

$$
C(\alpha, \beta)=\varlimsup_{t \rightarrow 0} \frac{1}{|t|}\left\|\alpha_{t}-\beta_{t}\right\| .
$$


We note that

$$
\begin{aligned}
\frac{1}{|s|}\left\|\alpha_{s}-\beta_{s}\right\|= & \frac{1}{n\left|\frac{s}{n}\right|}\left\|\alpha_{n \cdot \frac{s}{n}}-\beta_{n \cdot \frac{s}{n}}\right\| \\
& \leqq \frac{1}{n\left|\frac{s}{n}\right|} \sum_{k=1}^{n}\left\|\alpha_{\frac{s}{n}}^{n-k}\left(\alpha_{\frac{s}{n}}-\beta_{\frac{s}{n}}\right) \beta_{\frac{s}{n}}^{k-1}\right\| \\
& \leqq \frac{1}{\left|\frac{s}{n}\right|}\left\|\alpha_{\frac{s}{n}}-\beta_{\frac{s}{n}}\right\| .
\end{aligned}
$$

Thus $\left\|\alpha_{t}-\beta_{t}\right\|=O(t)$ if and only if $C(\alpha, \beta)<\infty$ and in that case

$$
\left\|\alpha_{t}-\beta_{t}\right\| \leqq C(\alpha, \beta)|t|,
$$

where $C(\alpha, \beta)$ is the best possible constant.

Theorem 3.1. Let $\mathscr{M}$ be a von Neumann algebra; $\alpha_{t}, \beta_{t} \sigma$-weakly continuous one parameter *automorphism groups of $\mathscr{M}$ with generators $\delta_{\alpha}, \delta_{\beta}$, respectively.

The following conditions are equivalent:

1. $\left\|\alpha_{t}-\beta_{t}\right\|=O(t)$.

2. $\mathscr{D}\left(\delta_{\alpha}\right)=\mathscr{D}\left(\delta_{\beta}\right)$ and there exists a bounded derivation $\delta$, of $\mathscr{M}$, such that

$$
\delta_{\alpha}(x)-\delta_{\beta}(x)=\delta(x) \quad \text { for all } \quad x \in \mathscr{D}\left(\delta_{\alpha}\right)=\mathscr{D}\left(\delta_{\beta}\right) \text {. }
$$

In this case $\|\delta\|=C(\alpha, \beta)$.

If the above conditions are satisfied and $\alpha_{t}$ is implemented by a strongly continuous group of unitaries, $U_{t}$, there exists a strongly continuous unitary group $V_{t}$, implementing $\beta_{t}$ such that

$$
\left\|U_{t}-V_{t}\right\| \leqq \frac{1}{2} C(\alpha, \beta)|t| .
$$

\section{Proof. $1 . \Rightarrow 2$.}

Theorem 2 of [14] establishes that $\mathscr{D}\left(\delta_{\alpha}\right)=\mathscr{D}\left(\delta_{\beta}\right)$ and that $\delta_{\alpha \beta} \equiv \delta_{\alpha}-\delta_{\beta}$ is a bounded operator from $\mathscr{D}\left(\delta_{\alpha}\right)$ into $\mathscr{M}$ with bound $C(\alpha, \beta)$. However the norm closure of $\mathscr{D}\left(\delta_{\alpha}\right)$, say $\mathfrak{A}$, is a $C^{*}$-algebra which is $\sigma$-weakly dense in $\mathscr{M}$. By the norm continuity of $\delta_{\alpha \beta}$ it extends to a bounded derivation of this $C^{*}$-algebra into $\mathscr{M}$. It is then a result of Kadison, Lemma 3 of ([22] also see [11]), that $\delta_{\alpha \beta}$ has a unique ultraweakly continuous extension, $\delta$, to $\mathscr{M}$. By the Kaplansky density theorem, the norm of this extension is also $C(\alpha, \beta)$. Note that although Kadison's lemma is stated for a derivation of a $C^{*}$-algebra into itself, the proof of the lemma does not rely in this fact.

2. $\Rightarrow 1$.

For $x \in \mathscr{D}\left(\delta_{\alpha}\right)=\mathscr{D}\left(\delta_{\beta}\right)$ we have

$$
\left(\alpha_{t}-\beta_{t}\right)(x)=\int_{0}^{t} d s \alpha_{s}\left(\delta_{a}-\delta_{\beta}\right) \beta_{t-s}(x)
$$


and so

$$
\left\|\left(\alpha_{t}-\beta_{t}\right)(x)\right\| \leqq|t|\|\delta\|\|x\| .
$$

One then has $\left\|\alpha_{t}-\beta_{t}\right\|=O(t)$ by applying the Kaplansky density theorem.

To complete the proof of the theorem we note that by the derivation theorem, [15], there exists an $h=h^{*} \in \mathscr{M}$ with $\|h\| \leqq \frac{1}{2}\|\delta\|=\frac{1}{2} C(\alpha, \beta)$ and such that

$$
\delta(x)=[i h, x], \quad x \in \mathscr{M} .
$$

If $U_{t}=e^{i t H_{\alpha}}$, then, [4],

$$
\delta_{\alpha}(x)=\left[i H_{\alpha}, x\right], \quad x \in \mathscr{D}\left(\delta_{\alpha}\right) .
$$

Thus

$$
\delta_{\beta}(x)=\left[i H_{\alpha}-i h, x\right], \quad x \in \mathscr{D}\left(\delta_{\beta}\right)=\mathscr{D}\left(\delta_{\alpha}\right) .
$$

Defining $V_{t} \equiv e^{i t\left(H_{\alpha}-h\right)}$, it follows by the Trotter product formula [18], that $V_{t} \mathscr{M} V_{t}^{*}=\mathscr{M}$. Thus, on defining $\beta_{t}^{\prime}(x)=V_{t} x V_{t}^{*}$ it is clear that $\delta_{\beta^{\prime}}=\delta_{\beta}$, so that $\beta^{\prime}=\beta$. Finally

$$
\left\|U_{t}-V_{t}\right\| \leqq|t|\|i h\|=|t| \frac{1}{2} C(\alpha, \beta) .
$$

We take a closer look at the situation of Theorem 3.1, when $\mathscr{M}$ is in standard form, $[8,1,7,6]$.

For a given faithful, normal, semifinite, weight on $\mathscr{M}$, let $\mathscr{P}^{\natural}$ be the natural cone, associated with $\mathscr{M}$ and $J$ the corresponding modular conjugation. Every vector $\eta \in \mathscr{H}$ can be written uniquely as $\eta=\eta_{1}-\eta_{2}+i\left(\eta_{3}-\eta_{4}\right)$ where $\eta_{i} \in \mathscr{P}^{\natural}$ and $\eta_{1} \perp \eta_{2}, \eta_{3} \perp \eta_{4}$. Moreover there exists a bijection $\zeta$ from the normal positive functionals $\varrho$ on $\mathscr{M}$ to vectors in $\mathscr{P}^{\natural}$ such that

$$
\varrho(x)=\omega_{\zeta(\varrho)}(x) \text { for } x \in \mathscr{M} .
$$

We have the inequality

$$
\left\|\zeta\left(\varrho_{1}\right)-\zeta\left(\varrho_{2}\right)\right\|^{2} \leqq\left\|\varrho_{1}-\varrho_{2}\right\| \leqq\left\|\zeta\left(\varrho_{1}\right)-\zeta\left(\varrho_{2}\right)\right\|\left\|\zeta\left(\varrho_{1}\right)+\zeta\left(\varrho_{2}\right)\right\| .
$$

For any $\sigma$-weakly continuous representation of a topological group $G$, as *-automorphisms of $\mathscr{M}$, there exists a strongly continuous representation $g \rightarrow U_{g}$ of $G$, uniquely determined by the requirements :

$$
\begin{aligned}
\alpha_{g}(x) & =U_{g} x U_{g}^{*} & & x \in \mathscr{M}, \quad g \in G \\
U_{g} \mathscr{P}^{\natural} & =\mathscr{P}^{\natural}, & & g \in G .
\end{aligned}
$$

This "canonical" representation is defined by

$$
U_{g} \zeta(\varrho)=\zeta\left(\alpha_{g-1}^{*} \varrho\right) \text {. }
$$

Given two representations $\alpha, \beta$ of $G$, if $U, V$ are the canonical unitaries described above, one easily sees that

$$
\frac{1}{2}\left\|U_{g}-V_{g}\right\|^{2} \leqq\left\|\alpha_{g^{-1}}-\beta_{g^{-1}}\right\| \leqq 2\left\|U_{g}-V_{g}\right\| .
$$

In particular if $\varphi$ is a faithful normal state on $\mathscr{M}$, then $\Delta_{\zeta(\varrho)}^{i t}$ is the canonical unitary group associated to the modular automorphism group of $\varphi$. 
When $G=R$ the next theorem states that the estimate $\frac{1}{2}\left\|U_{g}-V_{g}\right\|^{2}$ $\leqq\left\|\alpha_{g^{-1}}-\beta_{g^{-1}}\right\|$ can be improved.

Theorem 3.2 ${ }^{1}$. Let $\left\{\mathscr{M}, \mathscr{P}^{\natural}\right\}$ be a von Neumann algebra in standard form and $J$ the modular conjugation associated to this pair. If $\alpha_{t}, \beta_{t}$ are $\sigma$-weakly continuous one-parameter *-automorphism groups of, let $U_{t}=e^{i t H_{\alpha}}, V_{t}=e^{i t H_{\beta}}$ be the canonical unitaries, described above, which implement $\alpha_{t}, \beta_{t}$ respectively. The following are equivalent:

1. $\left\|\alpha_{t}-\beta_{t}\right\|=O(t)$.

2. $\left\|U_{t}-V_{t}\right\|=O(t)$.

3. There exists an element $h=h^{*} \in \mathscr{M}$ such that

$$
H_{\alpha}=H_{\beta}+h-J h J \text {. }
$$

Furthermore $\left\|U_{t}-V_{t}\right\| \leqq C(\alpha, \beta)|t|$.

Proof. 3. $\Rightarrow 2 . \Rightarrow 1$. are evident from Theorem 4 and its proof.

To show that $1 . \Rightarrow 3$. we observe that Theorem 4 and the derivation theorem allow us to find $h=h^{*} \in \mathscr{M}$ with $\|h\|=\frac{1}{2} C(\alpha, \beta)$ and

$$
\delta_{\alpha}(x)-\delta_{\beta}(x)=[i h, x], \quad x \in \mathscr{D}\left(\delta_{\alpha}\right)=\mathscr{D}\left(\delta_{\beta}\right) .
$$

Define a self-adjoint operator $H$ on $\mathscr{D}\left(H_{\beta}\right)$ by

$$
H=H_{\beta}+h-J h J \text {. }
$$

As $J h J \in \mathscr{M}^{\prime}$, the reasoning of Theorem 4 gives

$$
\alpha_{t}(x)=e^{i t \boldsymbol{H}} x e^{-i t \boldsymbol{H}}, \quad x \in \mathscr{M}, \quad t \in \boldsymbol{R}
$$

and

$$
\left\|e^{i t H}-e^{i t H_{\beta}}\right\| \leqq|t|\|h-J h J\| \leqq|t| C(\alpha, \beta) .
$$

It remains to show that $e^{i t H}$ is the canonical unitary group for $\alpha_{t}\left(\right.$ i.e. $H_{\alpha}=H$ ). For this we need to show that $e^{i t H} \mathscr{P}^{\natural} \subseteq \mathscr{P}^{\natural}$. However we know that $y J y J \mathscr{P}^{\natural} \subseteq \mathscr{P}^{\natural}$ for all $y \in \mathscr{M}$. Thus, by the Trotter product formula, if $\xi \in \mathscr{P}^{\natural}$, we have

$$
e^{i t(h-J h J)} \xi=\lim _{n \rightarrow \infty}\left[e^{\frac{t}{n} i h} J e^{\frac{t}{n} i h} J\right]^{n} \xi \in \mathscr{P}^{\natural} .
$$

\section{Writing}

$$
e^{i t\left(H_{\beta}+h-J h J\right)}=\text { strong limit }\left[\mathrm{e}^{\frac{i t}{n} H_{\beta}} e^{i \frac{t}{n}(h-J h J)}\right]^{n}
$$

we see that $e^{i t H} \mathscr{P}^{\natural}=\mathscr{P}^{\natural}$.

Remark. In particular we see from Theorem 5, that the Araki's perturbation [3] of the modular automorphism group [1] is characterized by $O(t)$ behaviour of the related modular automorphism groups.

1 This result has been improved by U. Haagerup who has shown that the spectrum of any automorphism is identical to the spectrum of the canonical implementing unitary $U(\alpha)$. Hence $\|U(\alpha)-U(\beta)\|=\|\alpha-\beta\|$ if $\|\alpha-\beta\|<2$ and $\|U(\alpha)-U(\beta)\| \geqq \sqrt{3}$ if $\|\alpha-\beta\|=2$ 
The rest of this section is devoted to the examination of groups of automorphisms which are close, but not necessarily of order $t$. First we need two results on cross-sections and cocycles.

Proposition 3.3. Let $\alpha_{t}, \beta_{t}$ be weakly continuous automorphism groups of a von Neumann algebra with separable pre-dual. Suppose that $\left\|\alpha_{t}-\beta_{t}\right\| \leqq \varepsilon<2$ for $|t|<\delta, \delta>0$. There then exists a Borel mapping $t \rightarrow U_{t}$ from $\boldsymbol{R}$ into the unitary group, $\mathscr{U}(\mathscr{M})$, of $\mathscr{M}$ such that $\beta_{t}(x)=U_{t} \alpha_{t}(x) U_{t}^{*}$ for all $t \in \boldsymbol{R}$ and

$$
\left\|U_{t}-I\right\| \leqq \varepsilon^{\prime} \quad \text { for } \quad|t| \leqq \delta \text {, where }\left(\varepsilon^{\prime}\right)^{2}=2\left(1-\sqrt{\frac{1-\varepsilon^{2}}{4}}\right) .
$$

Proof. An application of a result of Kadison and Ringrose [11] shows that $\gamma_{t}=\alpha_{t} \circ \beta_{-t}$ is inner for $|t| \leqq \delta$ and hence, by the cocycle identity for $\gamma_{t}$, for all $t \in \boldsymbol{R}$. Furthermore Lemma 5 of [11] shows that for each $t,|t| \leqq \delta$ we can choose the unitary so that $\left\|U_{t}-I\right\|^{2} \leqq 2\left(1-\sqrt{1-\frac{\left\|\gamma_{t}-i\right\|^{2}}{4}}\right)=\left(\varepsilon^{\prime}\right)^{2}$, and this is the minimal possible norm of $U_{t}-I$.

We must now examine the Borel structure in the inner automorphism group, $\operatorname{Inn}(\mathscr{M})$. Indeed what we need is a variation on the proof of Theorem 4.13 of [29]. Recall that a topological space is called Polish if it is homeomorphic to a complete separable metric space. A subset of a Polish space (see [27]) is analytic if it is a continuous image of a Polish space and if $X$ and $Y$ are analytic Borel spaces and $f$ is a 1-1 Borel map of $X$ onto $Y$, then $f$ is a Borel isomorphism [27,p. 72]. Consider $\mathscr{U}(\mathscr{M})$, with the strong *-topology and $\mathscr{U}(\mathscr{Z})$, the closed subgroup of $\mathscr{U}(\mathscr{M})$, consisting of the unitaries in the center $\mathscr{Z}=\mathscr{M} \cap \mathscr{M}^{\prime}$. Let $\mathscr{B}\left(\mathscr{M}_{*}\right)$ denote the bounded operators on $\mathscr{M}_{*}$ with the topology of pointwise norm convergence. Since $\mathscr{M}_{*}$ is separable, $\mathscr{U}(\mathscr{M})$ and $\mathscr{B}\left(\mathscr{M}_{*}\right)$ are Polish. Moreover, the subset $\operatorname{Inn}(\mathscr{M}) \subseteq \mathscr{B}\left(\mathscr{M}_{*}\right)$ is analytic, since the canonical map $\mathscr{U}(\mathscr{M}) \rightarrow \operatorname{Inn}(\mathscr{M})$ is continuous. The space $\operatorname{Inn}(\mathscr{M})$ will be the space $Y$ in the result quoted above. The space $\mathscr{U}(\mathscr{M}) / \mathscr{U}(\mathscr{Z})$ is to be $X$ and $f$ is the $1-1$ continuous map of an element in $\mathscr{U}(\mathscr{M}) / \mathscr{U}(\mathscr{Z})$ to the automorphism it induces. We conclude that the Borel structure of $\operatorname{Inn}(\mathscr{M})$ is the same as that of $\mathscr{U}(\mathscr{M}) / \mathscr{U}(\mathscr{Z})$.

Since the quotient map $\eta: \mathscr{U}(\mathscr{M}) \rightarrow \mathscr{U}(\mathscr{M}) / \mathscr{U}(\mathscr{Z})$ is continuous and open, it follows that the canonical map $f \circ \eta: \mathscr{U}(\mathscr{M}) \rightarrow \operatorname{Inn}(\mathscr{M})$ is continuous and maps open sets into Borel sets. As $\mathscr{U}(\mathscr{M})$ is a Polish space it follows from Theorem 3.4.1 of [27] that $f \circ \eta$ admits a Borel cross section, i.e. there exists a Borel map $U: \operatorname{Inn}(\mathscr{M}) \rightarrow \mathscr{U}(\mathscr{M})$ such that

$$
\alpha(x)=U(\alpha) x U(\alpha)^{*}
$$

for all $\alpha \in \operatorname{Inn}(\mathscr{M}), x \in \mathscr{M}$. The existence of $t \rightarrow U_{t}$ for $|t|>\delta$ follows immediately.

For $|t| \leqq \delta$ we proceed by defining

$$
\begin{aligned}
\mathscr{U}_{\varepsilon^{\prime}}(\mathscr{M}) & =\left\{U \in \mathscr{U}(\mathscr{M}) ;\|U-I\| \leqq \varepsilon^{\prime}\right\} \\
\operatorname{Inn}_{\varepsilon}(\mathscr{M}) & =\{\alpha \in \operatorname{Inn}(\mathscr{M}) ;\|\alpha-i\| \leqq \varepsilon\} .
\end{aligned}
$$

The image of $\mathscr{U}_{\varepsilon^{\prime}}(\mathscr{M})$ in $\operatorname{Inn}(\mathscr{M})$ is just $\operatorname{Inn}_{\varepsilon}(\mathscr{M})$ by the Kadison-Ringrose theorem. $\mathscr{U}_{\varepsilon^{\prime}}(\mathscr{M})$ is closed in $\mathscr{U}(\mathscr{M})$, hence Polish, and furthermore $\mathscr{U}_{\varepsilon^{\prime}}(\mathscr{M})$ is a $G_{\delta}$ set in 
$\mathscr{U}(\mathscr{M})$, thus the image of $\mathscr{U}_{\varepsilon^{\prime}}(\mathscr{M})$ in $\mathscr{U}(\mathscr{M}) / \mathscr{U}(\mathscr{Z})$ is Borel. As the open sets in $\mathscr{U}_{\varepsilon^{\prime}}(\mathscr{M})$ are intersections of open sets in $\mathscr{U}(\mathscr{M})$ with $\mathscr{U}_{\varepsilon^{\prime}}(\mathscr{M})$, it follows that $f \circ \eta$, restricted to $\mathscr{U}_{\varepsilon^{\prime}}(\mathscr{M})$, maps open sets in $\mathscr{U}_{\varepsilon^{\prime}}(\mathscr{M})$ into Borel sets in $\operatorname{Inn}(\mathscr{M})$. Another application of Theorem 3.4.1 of [27] on $f \circ \eta: \mathscr{U}_{\varepsilon^{\prime}}(\mathscr{M}) \rightarrow \operatorname{Inn}_{\varepsilon}(\mathscr{M})$ implies the existence of a Borel cross section for $|t| \leqq \delta$.

We next prove an implementabiliary theorem for inner cocycles on von Neumann algebras. Related results abound in the literature. Suppose $\mathscr{M}$ is a von Neumann algebra with separable pre-dual $\mathscr{M}_{*}$ and $t \rightarrow \sigma_{t}=\beta_{t} \cdot \alpha_{-t}$ is a $\sigma$-weakly continuous pointwise inner cocycle in the automorphism group of $\mathscr{M}$. It was shown in [24, Theorem 1.2.8] that $\sigma_{t}$ is unitarily implementable if $\mathscr{M}$ is a factor, and in [28, Theorem 5.3] this result is extended to arbitrary $\mathscr{M}$ if $\alpha$ is centrefixing. If $\lim _{t \rightarrow 0}\left\|\sigma_{t}-\imath\right\|=0$, then in [5, Proposition 4.1] it is shown that there exists a norm continuous unitary cocycle implementing $\sigma$. This last result holds without the separability of $\mathscr{M}_{*}$. Some features of these results are evident in what follows.

Theorem 3.4. Let $\mathscr{M}$ be a von Neumann algebra with separable predual and let $\alpha, \beta$ be two $\sigma$-weakly continuous oneparameter groups of automorphisms of $\mathscr{M}$. Assume there exists $\delta, \varepsilon>0$ such that $\varepsilon<\sqrt{\frac{71}{18}} \cong 0.47$ and

$$
\left\|\alpha_{t}-\beta_{t}\right\| \leqq \varepsilon \text { when }|t| \leqq \delta .
$$

Then there exists a $\sigma$-weakly continuous cocycle $t \rightarrow \Gamma_{t}$ in $\mathscr{U}(\mathscr{M})$ such that

$$
\begin{aligned}
& \Gamma_{t+s}=\Gamma_{t} \alpha_{t}\left(\Gamma_{s}\right) \quad \text { for } t, s \in \boldsymbol{R} \\
& \beta_{t}(x)=\Gamma_{t} \alpha_{t}(x) \Gamma_{t}^{*} \text { for } \quad t \in \boldsymbol{R}, \quad A \in \mathscr{M} \\
& \left\|\Gamma_{t}-I\right\| \leqq 10 \sqrt{2\left(1-\sqrt{1-\frac{\varepsilon^{2}}{4}}\right)}=5 \varepsilon+0\left(\varepsilon^{2}\right) \quad \text { for } \quad|t|<\frac{\delta}{4} .
\end{aligned}
$$

Proof. The proof goes by standard cohomological considerations as in [5]. First note by Proposition 3.3 that there exists a Borel map $t \in R \rightarrow U_{t} \in \mathscr{U}(\mathscr{M})$ such that

$$
\beta_{t}(x)=U_{t} \alpha_{t}(x) U_{t}^{*}, \quad A \in \mathscr{M}, \quad t \in \boldsymbol{R}
$$

and

$$
\left\|U_{t}-I\right\| \leqq \varepsilon^{\prime}
$$

for $|t| \leqq \delta$, where $\varepsilon^{\prime 2}=2\left(1-\sqrt{1-\frac{\varepsilon^{2}}{4}}\right)$. Now, define

$$
z(s, t)=U_{s} \alpha_{s}\left(U_{t}\right) U_{s+t}^{-1} .
$$

Then $z: \boldsymbol{R}^{2} \rightarrow \mathscr{U}\left(\mathscr{M} \cap \mathscr{M}^{\prime}\right)$ is a centre-valued two-cocycle, i.e. $z(s, 0)=z(0, t)=I$ for all $s$ and $t$ and

$$
z(s, t) z(s+t, u)=\alpha_{s}(z(t, u)) z(s, t+u)
$$

for all $s, t, u$. From the definition of $z$ we immediately get the estimate

$$
\|z(s, t)-I\| \leqq 3 \varepsilon^{\prime} \quad \text { for } \quad|s|+|t|<\delta .
$$


Define $t \in R \rightarrow \lambda_{t} \in \mathscr{U}(\mathscr{M} \cap \mathscr{M})$ inductively by $\lambda_{0}=I$ and

$$
\frac{\lambda \delta}{2}(t+n)=\frac{\lambda \delta}{2} n z\left(\frac{\delta}{2} n, \frac{\delta}{2} t\right)
$$

for $0 \leqq t \leqq 1, n \in \boldsymbol{Z}$.

Next, define $z^{\prime}(s, t) \equiv \lambda_{s} \alpha_{s}\left(\lambda_{t}\right) z(s, t) \lambda_{s+t}^{-1}$ for $s, t \in \boldsymbol{R}$. By [5], Lemma 3.3, $z^{\prime}(s, t)$ is a 2-cocycle satisfying

$$
z^{\prime}(p, t)=I \quad \text { for } \quad 0 \leqq t \leqq \frac{\delta}{2}, p \in \frac{\delta}{2} Z
$$

and

$$
\left\|z^{\prime}(s, t)-I\right\| \leqq 6 \varepsilon^{\prime} \quad \text { for } \quad 0 \leqq s, t \leqq \frac{\delta}{2} .
$$

By [5], Lemma 3.2a;s $s \rightarrow \alpha_{-s}\left(z^{\prime}(s, t)\right)$ is periodic with period $\frac{\delta}{2}$, and hence the estimate on $z^{\prime}(s, t)-I$ just derived extends to all $s$. Now, let log denote the principal value of the logarithm on the complex plane with a cut along the negative real axis. Now, since $\varepsilon<\sqrt{\frac{71}{18}}$ it follows that $6 \varepsilon^{\prime}<2$, and hence by the estimate on $z^{\prime}$, we can consistently define $y(s, t)=\log \left(z^{\prime}(s, t)\right)$ for $0 \leqq t<\frac{\delta}{2}$, and the cocycle property of $z^{\prime}(s, t)$ gives

$$
\begin{aligned}
& y(s, 0)=y(0, t)=0 \\
& y(s, t)+y(s+t, u)=\alpha_{s}(y(t, u))+y(s, t+u)
\end{aligned}
$$

for $0 \leqq t, u, t+u \leqq \frac{\delta}{2}$. Also $s, t \rightarrow y(s, t)$ is a Borel map. Proceeding as in [5], proof of Proposition 3.5, we define a Borel map $c: t \in\left[0, \frac{\delta}{2}\right] \rightarrow c_{t} \in \mathscr{M} \cap \mathscr{M}^{\prime}$ by

$$
c_{t}=-\frac{2}{\delta} \int_{0}^{\frac{\delta}{2}} d s \alpha_{-s}(y(s, t))
$$

and $\lambda^{\prime}: t \in\left[0, \frac{\delta}{2}\right] \rightarrow \lambda_{t}^{\prime} \in \mathscr{U}\left(\mathscr{M} \cap \mathscr{M}^{\prime}\right)$ by

$$
\lambda_{t}^{\prime}=\exp \left(c_{t}\right)
$$

and compute that

$$
z^{\prime}(s, t)=\lambda_{s+t}^{\prime} \alpha_{s}\left(\lambda_{t}^{\prime-1}\right) \lambda_{s}^{\prime-1}
$$

for $0 \leqq s, t, s+t \leqq \frac{\delta}{2}$. Using the spectral radius formula for unitaries and selfadjoints and the definition of $\lambda_{t}^{\prime}$ from $z^{\prime}(s, t)$ by means of $y(s, t)$ and $c_{t}$ we 
immediately derive the estimates

$$
\|y(s, t)\| \leqq \arccos \left(1-\frac{\left(6 \varepsilon^{\prime}\right)^{2}}{2}\right) ; \quad 0 \leqq t \leqq \frac{\delta}{2}
$$

thus

$$
\left\|c_{t}\right\| \leqq \arccos \left(1-\frac{\left(6 \varepsilon^{\prime}\right)^{2}}{2}\right) ; \quad 0 \leqq t \leqq \frac{\delta}{2}
$$

and finally

$$
\left\|\lambda_{t}^{\prime}-I\right\| \leqq 6 \varepsilon^{\prime}
$$

for $0 \leqq t \leqq \frac{\delta}{2}$. We now extend the map $t \in\left[0, \frac{\delta}{2}\right] \rightarrow \lambda_{t}^{\prime} \in \mathscr{U}\left(\mathscr{M} \cap \mathscr{M}^{\prime}\right)$ to a Borel map of all of $\boldsymbol{R}$ and define

$$
z^{\prime \prime}(s, t) \equiv \lambda_{s+t}^{\prime-1} z^{\prime}(s, t) \lambda_{s}^{\prime} \alpha_{s}\left(\lambda_{t}^{\prime}\right) .
$$

Then $z^{\prime \prime}$ is a 2-cocycle, and

$$
z^{\prime \prime}(s, t)=I
$$

for $0 \leqq s, t \leqq \frac{\delta}{4}$.

Now, replacing $\frac{\delta}{2}$ by $\frac{\delta}{4}$ and $\varepsilon^{\prime}$ by 0 , we define $z^{\prime \prime \prime}$ from $z^{\prime \prime}$ as $z^{\prime}$ was defined from $z$. First define

$$
\begin{aligned}
& \lambda_{0}^{\prime \prime}=I \\
& \lambda_{\frac{\delta}{4}(t+n)}^{\prime \prime}=\lambda_{\frac{\delta}{4} n}^{\prime \prime} z^{\prime \prime}\left(\frac{\delta}{4} n, \frac{\delta}{4} t\right) ; \quad 0 \leqq t \leqq 1, \quad n \in \boldsymbol{Z}
\end{aligned}
$$

and then

$$
z^{\prime \prime \prime}(s, t)=\lambda_{s}^{\prime \prime} \alpha_{s}\left(\lambda_{t}^{\prime \prime}\right) z^{\prime \prime}(s, t) \lambda_{s+t}^{\prime \prime}+1
$$

By [5], Lemma 3.3, $z^{\prime \prime \prime}$ is a 2-cocycle such that

$$
z^{\prime \prime \prime}(p, t)=I \quad \text { for } \quad 0 \leqq t \leqq \frac{\delta}{4}, \quad p \in \frac{\delta}{4} Z
$$

and

$$
z^{\prime \prime \prime}(s, t)=I \quad \text { for } \quad 0 \leqq s, t \leqq \frac{\delta}{4} .
$$

Hence [5], Lemma 3.2b implies that $z^{\prime \prime \prime}(s, t)=I$ for all $s, t$. Defining $\lambda_{s}^{\prime \prime \prime}=\lambda_{s}^{\prime \prime} \lambda_{s}^{\prime} \lambda_{s}$ we thus have

$$
\begin{aligned}
z(s, t) & =\lambda_{s+t}^{\prime \prime \prime} z^{\prime \prime \prime}(s, t) \lambda_{s}^{\prime \prime \prime}-1 \alpha_{s}\left(\lambda_{t}^{\prime \prime \prime}-1\right) \\
& =\lambda_{s+t}^{\prime \prime \prime} \lambda_{s}^{\prime \prime \prime}-1 \alpha_{s}\left(\lambda_{t}^{\prime \prime \prime}-1\right) .
\end{aligned}
$$


Define

$$
\Gamma_{s}=\lambda_{s}^{\prime \prime \prime} U_{s} .
$$

Then by the definition of $z$ from $U, \Gamma_{s}$ is a two-cocycle:

$$
\Gamma_{s+t}=\Gamma_{s} \alpha_{s}\left(\Gamma_{t}\right)
$$

and

$$
\beta_{t}(x)=\Gamma_{t} \alpha_{t}(x) \Gamma_{t}^{*} .
$$

$t \rightarrow \Gamma_{t}$ is Borel, hence strongly continuous by [24], proof of Lemma 1.2.5. [We note the following brief proof. If $\mathscr{M}$ is in standard form, let $\zeta$ be the canonical map of $\mathscr{M}_{*+} \rightarrow \mathscr{P}^{\natural}[10]$. As $\mathscr{M}_{*+}$ contains a norm-dense subsequence it follows from the estimate $\left\|\zeta\left(\omega_{1}\right)-\zeta\left(\omega_{2}\right)\right\|^{2} \leqq\left\|\omega_{1}-\omega_{2}\right\|$ that $\mathscr{P}^{\natural}$ contains a norm dense subsequence. Then $\mathscr{H}=\mathscr{P}^{\natural}-\mathscr{P}^{\natural}+i\left(\mathscr{P}^{\natural}-\mathscr{P}^{\natural}\right)$ is separable. If $s \rightarrow V_{s}$ is the canonical unitary implementation of $\alpha$ on $\mathscr{H}$ put $U_{s}=\Gamma_{s} V_{s}$, and note that $s \rightarrow U_{s}$ is a Borel map and a unitary representation of $\boldsymbol{R}$ (implementing $\beta$ ). As $\mathscr{H}$ is separable it follows that $s \rightarrow U_{s}$ is strongly continuous so then $s \rightarrow \Gamma_{s}=U_{s} V_{-s}$ is strongly continuous.] We have the estimates

$$
\begin{array}{rll}
\left\|U_{t}-I\right\| \leqq \varepsilon^{\prime} & \text { for } & |t| \leqq \delta \\
\left\|\lambda_{t}-I\right\| \leqq 3 \varepsilon^{\prime} & \text { for } & 0 \leqq t \leqq \frac{\delta}{2} \\
\left\|\lambda_{t}^{\prime}-I\right\| \leqq 6 \varepsilon^{\prime} & \text { for } & 0 \leqq t \leqq \frac{\delta}{2} \\
\left\|\lambda_{t}^{\prime \prime}-I\right\|=0 & \text { for } & 0 \leqq t \leqq \frac{\delta}{4}
\end{array}
$$

and hence

$$
\left\|\Gamma_{s}-I\right\| \leqq \varepsilon^{\prime}+3 \varepsilon^{\prime}+6 \varepsilon^{\prime}=10 \varepsilon^{\prime}
$$

for $|s| \leqq \frac{\delta}{4}$.

Now we apply these result to the analysis of automorphisms which are close for all $t$ thus generalizing a result of Reynolds [26] and Theorem 2.4.

Theorem 3.5. Let $\mathscr{M}$ be a von Neumann algebra with separable predual, and let $\alpha, \beta$ be $\sigma$-weakly continuous one parameter groups of *-automorphisms of $\mathscr{M}$. Assume there exists $a 0 \leqq \varepsilon \sqrt{\frac{199}{50}} \cong 0.28$ such that

$$
\left\|\alpha_{t}-\beta_{t}\right\| \leqq \varepsilon
$$

for all $t$.

There exists an inner automorphism $\gamma$ of $\mathscr{M}$ such that $\alpha_{t}=\gamma \circ \beta_{t} \circ \gamma^{-1}$ 
We can choose a unitary $W \in \mathscr{M}$, giving $\gamma$, so that $\|W-I\| \leqq 10 \sqrt{2\left(1-\sqrt{1-\frac{\varepsilon^{2}}{4}}\right)}$.

Thus $\|\gamma-\imath\| \leqq 10 \varepsilon+0\left(\varepsilon^{2}\right)$.

Proof. By Theorem 3.4 there exists a strongly continuous unitary cocycle $t \rightarrow \Gamma_{t}$ in $\mathscr{U}(\mathscr{M})$ such that

$$
\begin{aligned}
& \Gamma_{t+s}=\Gamma_{t} \beta_{t}\left(\Gamma_{s}\right) \\
& \alpha_{t}(x)=\Gamma_{t} \beta_{t}(x) \Gamma_{t}^{*}
\end{aligned}
$$

for all $x \in \mathscr{M}, t, s \in \boldsymbol{R}$, and such that $\left\|\Gamma_{t}-I\right\| \leqq \varepsilon^{\prime}=10 \sqrt{2\left(1-\sqrt{1-\varepsilon^{2}} / 4\right)}<\sqrt{2}$ for all $t$.

We may assume that $\mathscr{M}$ is in a standard representation, hence there exists a strongly continuous unitary group $t \rightarrow V_{t}$ such that

$$
\beta_{t}(x)=V_{t} x V_{t}^{*}
$$

for $x \in \mathscr{M}, t \in \boldsymbol{R}$ [10]. Define $U_{t}=\Gamma_{t} V_{t}$. Then $U_{s} U_{t}=\Gamma_{s} V_{s} \Gamma_{t} V_{t}=\Gamma_{s} \beta_{s}\left(\Gamma_{t}\right) V_{s+t}$ $=\Gamma_{s+t} V_{s+t}=U_{s+t}$, so $t \rightarrow U_{t}$ is a strongly continuous unitary group, and

$$
\alpha_{t}(x)=U_{t} x U_{t}^{*}
$$

for $x \in \mathscr{M}, t \in \boldsymbol{R}$. Since $U_{t} V_{-t}=\Gamma_{t} \in \mathscr{M}$, it follows from Theorem 2.4 that there exists a unitary $W \in \mathscr{M}$ such that $U_{t}=W V_{t} W^{*}$. Defining $\gamma(x)=W x W^{*}$ we thus have

$$
\alpha_{t}=\gamma \circ \beta_{t} \circ \gamma^{-1}
$$

for all $t \in \boldsymbol{R}$.

The estimate on $\|W-I\|$ is essentially that at the end of Theorem 2.2

Theorem 3.6. Let $\mathscr{M}$ be a von Neumann algebra with separable predual and let $\alpha, \beta$ be two $\sigma$-weakly continuous one-parameter groups of $*$-automorphism of $\mathscr{M}$ with generators $\delta_{\alpha}$, and $\delta_{\beta}$, respectively.

The following conditions are equivalent:

1. There exists $\varepsilon_{1}, 0 \leqq \varepsilon_{1}<\sqrt{199} / 50 \cong 0.28$ and $\delta_{1}>0$ such that

$$
\left\|\alpha_{t}-\beta_{t}\right\| \leqq \varepsilon_{1}
$$

for $0 \leqq t \leqq \delta_{1}$.

2. There exists $\varepsilon_{2}, 0 \leqq \varepsilon_{2} \leqq \sqrt{199} / 50$ and $\delta_{2}>0$, an inner automorphism $\gamma$ of $\mathscr{M}$, and a bounded derivation $\delta$ of $\mathscr{M}$ such that

$$
\delta_{\alpha}=\gamma \circ\left(\delta_{\beta}+\delta\right) \circ \gamma^{-1}
$$

and

$$
\left\|\alpha_{t} \circ \gamma^{-1} \circ \alpha_{-t} \circ \gamma-\imath\right\| \leqq \varepsilon_{2}
$$

for $0 \leqq t \leqq \delta_{2}$.

If these conditions are satisfied then

$$
\left\|\alpha_{t}-\beta_{t}\right\|=\left\|\alpha_{t} \circ \gamma^{-1} \circ \alpha_{-t} \circ \gamma-\imath\right\|+O(t) \text {. }
$$


Moreover, a $W \in \mathscr{M}$ can be chosen, giving $\gamma$, such that

$$
\|W-I\| \leqq 10 \sqrt{2\left(1-\sqrt{1-\frac{\varepsilon_{1}^{2}}{4}}\right)} .
$$

Thus $\|\gamma-\imath\| \leqq 10 \varepsilon_{1}+0\left(\varepsilon_{1}^{2}\right)$.

Proof. $1 \Rightarrow 2$. Proceeding as in the proof of the preceding theorem, we may assume that $\mathscr{M}$ is in a standard representation, and find strongly continuous unitary groups $U, V$ such that

$$
\begin{aligned}
& \alpha_{t}(x)=U_{t} x U_{t}^{*}, \beta^{t}(x)=V_{t} x V_{t}^{*} \\
& U_{t} V_{-t} \in \mathscr{M} \text { for } t \in \dot{R} \\
& \left\|U_{t}-V_{t}\right\| \leqq \varepsilon^{\prime}<\sqrt{2} \text { for }|t| \leqq \delta / 4 .
\end{aligned}
$$

In this case $\Omega \equiv \frac{4}{\delta} \int_{0}^{\delta / 4} d t U_{t} V_{-t} \in \mathscr{M}$. Hence by Theorem 2.2 there exists a unitary $W \in \mathscr{M}$ such that

$$
\left\|U_{t}-W V_{t} W^{*}\right\|=O(t) .
$$

Defining $\gamma(x)=W x W^{*}$ and $\hat{\beta}_{t}^{t}=\gamma^{\circ} \beta_{t} \circ \gamma^{-1}$ we then have

$$
\left\|\alpha_{t}-\hat{\beta}_{t}\right\| \leqq 2\left\|U_{t}-W V_{t} W^{*}\right\|=O(t) .
$$

Hence by Theorem 3.1 there exists a bounded derivation $\delta^{\prime}$ of $\mathscr{M}$ such that

$$
\begin{aligned}
\delta_{\alpha}=\delta_{\hat{\beta}}+\delta^{\prime} & =\gamma \circ \delta_{\beta} \circ \gamma^{-1}+\delta^{\prime} \\
& =\gamma \circ\left(\delta_{\beta}+\delta\right) \circ \gamma^{-1}
\end{aligned}
$$

where $\delta=\gamma^{-1} \circ \delta^{\prime} \circ \gamma$.

It follows immediately that

$$
\begin{aligned}
\alpha_{t} \circ \gamma^{-1} \circ \alpha_{t} \circ \gamma= & \exp \left(t \delta_{\alpha}\right)-\exp \left(t \delta_{\beta}\right) \\
& +\exp \left(t \delta_{\beta}\right)-\exp \left(t\left(\delta_{\beta}+\delta\right)\right)
\end{aligned}
$$

and hence

$$
\left\|\alpha_{t} \circ \gamma^{-1} \circ \alpha_{-t} \circ \gamma-\imath\right\|=\left\|\alpha_{t}-\gamma^{-1} \alpha_{t} \gamma\right\|=\left\|\alpha_{t}-\beta_{t}\right\|+O(t) .
$$

Hence the estimate in 2 follows from the estimate in 1 , and conversely the estimate in 1 follows from 2 .

The estimate of $\|W-I\|$ is by now clear.

\section{§4. Special Cases and Examples}

In Theorem 3.1 we saw an example where the twist occurring in Theorems 2.1, 2.2, 3.6 was not necessary. Other such cases are given in Propositions 4.1 and 4.3.

Proposition 4.1. Let $U_{t}, V_{t}$ be $C_{0}$ or $C_{0}^{*}$ groups of isometries on a Banach space $X$, with generators $S, T$ respectively. Assume that $U_{t} V_{s}=V_{s} U_{t}, s, t \in R$ and that there exists $\varepsilon>0$ such that $\left\|U_{t}-V_{t}\right\|<2$ for $|t| \leqq \varepsilon$. Then $\left\|U_{t}-V_{t}\right\|=O(t)$ and $\mathscr{D}(S)=\mathscr{D}(T)$. 
Moreover $T-S$ is bounded in norm and extends to a bounded operator on $X$ which is $\sigma\left(X, X_{*}\right)$ closed in the $C_{0}^{*}$-case.

Proof. Define $W_{t}=U_{t} V_{-t}$. Since $U$ and $V$ commute, $t \rightarrow W_{t}$ is a one parameter group of isometries of $X$. We claim $t \rightarrow W_{t} x$ is continuous in the appropriate topology. The $C_{0}$ case is trivial. For the $C_{0}^{*}$ case we observe that $U_{t}$ is the dual of $U_{t}^{*}$ acting in the predual of $X, X_{*}$. The same being true for $V_{t}$ one sees that $W_{t}$ is the dual group of the strongly continuous group $V_{-t}^{*} U_{t}^{*}$ and so is weak* continuous.

Thus $W_{t}$ is a continuous group of isometries such that $\left\|W_{t}-I\right\|<2$, for $|t| \leqq \varepsilon$. Let $\operatorname{Sp}(W)$ denote the spectrum of $W$ as defined in [23]. If $p \in \operatorname{Sp}(W)$ it follows [24, Lemma 2.36], that there exists a sequence $\left\{x_{n}\right\} \subseteq X$ such that $\left\|x_{n}\right\|=1$, for all $n$ and

$$
\lim _{n \rightarrow \infty}\left\|W_{t} x_{n}-e^{-i p t} x_{n}\right\|=0,
$$

uniformly for $t$ in a compact set. We have that $\left\|W_{t} x_{n}-x_{n}\right\| \geqq\left\|e^{-i p t} x_{n}-x_{n}\right\|$ $-\left\|W_{t} x_{n}-e^{-i p t} x_{n}\right\|$. Then $\left\|W_{t}-\imath\right\| \geqq\left|e^{-i p t}-1\right|$ for $p \in \operatorname{Sp}(W)$. However if $|t| \leqq \varepsilon$ and $p \in \operatorname{Sp}(W)$ we then have $\left|e^{-i p t}-1\right|<2$. Thus $|p| \leqq \pi / \varepsilon$, i.e. the spectrum of $W$ is bounded. Now by [25, Proposition 2.2], $t \rightarrow W_{t}$ is norm continuous and so has a bounded generator, continuous in the appropriate topology. As $U_{t}=W_{t} V_{t}$, the rest of the proposition follow easily.

Note that if $\varepsilon=\infty$ in Proposition 4.1, the proof shows that $\operatorname{Sp}(W)=\{0\}$. But then $W_{t}=\imath$ for all $t$ [23] and so we get the following strengthening of Theorems 2.4 and 3.5 in this special case.

Corollary 4.2. With the same notation and assumptions as in Proposition 11, if $\left\|U_{t}-V_{t}\right\|<2$ for all $t \in \boldsymbol{R}$, then $U_{t}=V_{t}$.

Another example where the twist of Theorem 3.6 is not necessary is for quasifree automorphism groups of the CAR algebra [13].

Given a separable complex Hilbert space $\mathscr{H}$, the CAR-algebra, $\mathfrak{U}(\mathscr{H})$, over $\mathscr{H}$ is the $C^{*}$-algebra generated by the identity and elements $a(f)$, where $f \rightarrow a(f)$ is a linear map of $\mathscr{H}$ satisfying the anti-commutation relations.

$$
\begin{aligned}
& a(f)^{*} a(g)+a(g) a(f)^{*}=(g, f) I \\
& a(f) a(g)+a(g) a(f)=0 .
\end{aligned}
$$

This $C^{*}$-algebra is a UHF $C^{*}$-algebra (more recent terminology is "uniformly matricial $C^{*}$-algebra) and is a fortiori simple.

The automorphism groups we study are quasi-free automorphism groups. Such a group arises as follows : Let $t \rightarrow U_{t}$ be a one parameter group of unitaries of the underlying Hilbert space.

Define

$$
\alpha_{t}(a(f))=a\left(U_{t} f\right) \text {. }
$$

One readily sees that $\alpha_{t}$ extends to an automorphism group of $\mathfrak{U}(\mathscr{H})$.

This algebra has been studied in great detail. We shall make use of the fact [13] that there exists a representation (the Fock representation) of $\mathfrak{U}(\mathscr{H})$ where every quasi-free automorphism group is unitarily implemented. 
The Fock state of the CAR-algebra is the unique state $\omega_{F}$ with the property that $\omega\left(a(f)^{*} a(g)\right)=0$. This is a pure state. As this defining relation is invariant under quasi-free automorphisms, so is the state and then applying the GNS procedure for the state $\omega_{F}$ we find a representation of $\mathfrak{U}, \pi_{\omega_{F}}$, a Hilbert space $\mathscr{H}_{\boldsymbol{F}}$ and unitaries $U^{\alpha}(t)$ on $\mathscr{H}_{F}$ such that

$$
\pi_{\omega_{F}}\left(\alpha_{t}(x)\right)=U^{\alpha}(t) \pi_{\omega_{F}}(x) U^{\alpha}(-t) .
$$

Araki's characterization, [2], of bounded derivations of $\mathfrak{U}(\mathscr{H})$ will play in a key role in the following theorem.

Theorem 4.3. Let $\mathfrak{A}=\mathfrak{U}(\mathscr{H})$ be the CAR algebra and suppose $\alpha_{t}, \beta_{t}$ are two quasifree automorphism groups of $\mathfrak{U}$ corresponding to unitary groups $U_{t}, V_{t}$ respectively. Let $H, K$ be the self-adjoint generators of $U_{t}, V_{t}$ respectively and write $\alpha_{t}=\exp \left(t \delta_{H}\right)$, $\beta_{t}=\exp \left(t \delta_{K}\right)$ for the appropriate closed derivations of $\mathfrak{U}$.

The following conditions are equivalent:

1. $\mathscr{D}(H)=\mathscr{D}(K)$ and $\|H-K\|_{\mathrm{Tr}}<\infty\left(\|\|_{\mathrm{Tr}}\right.$ denotes the trace class norm.

2. $\left\|U_{t}-V_{t}\right\|_{\mathrm{Tr}}=O(t), t \rightarrow 0$.

3. $\mathscr{D}\left(\delta_{H}\right)=\mathscr{D}\left(\delta_{K}\right)$ and $\left\|\delta_{H}-\delta_{K}\right\|<\infty$.

4. $\left\|\alpha_{t}-\beta_{t}\right\|=O(t), t \rightarrow 0$.

Proof. $1 . \Rightarrow 2$. We have the integral relation

$$
\frac{\left(U_{t}-V_{t}\right)}{t}=\frac{i}{t} \int_{0}^{t} d s U_{s}(H-K) V_{t-s} .
$$

Therefore,

$$
\left\|\left(U_{t}-V_{t}\right) / t\right\|_{\mathrm{Tr}} \leqq\|(H-K)\|_{\operatorname{Tr}} .
$$

2. $\Rightarrow 1$.

First we note that $\left\|\left(U_{t}-V_{t}\right)\right\|=O(t)$ where $\|\cdot\|$ denotes the usual operator norm on $\mathscr{H}$. Applying Corollary 3 of [14], we conclude that $\mathscr{D}(H)=\mathscr{D}(K)$, and $H-K$ has a bounded extension to all of $\mathscr{H}$. We write this extension as $H-K$. Let $D_{t}=\left(U_{t}-V_{t}\right) / t$ and suppose $\psi \in \mathscr{D}(H)=\mathscr{D}(K)$. Then

$$
\left(D_{t}-i(H-K)\right) \psi=\left(\frac{U_{t}-1}{t}-i H\right) \psi+\left(\frac{V_{t}-1}{t}-i K\right) \psi
$$

and so $D_{t}$ converges strongly to $i(H-K)$ as $t \rightarrow 0$. Since $D_{t}^{*}=D_{-t}$ converges to $-i(H-K)$ one has (the $D_{t}$ are uniformly bounded) that

$$
\left|D_{t}\right|^{2}=D_{t}^{*} D_{t} \rightarrow|H-K|^{2} \text { strongly . }
$$

Since the $\left|D_{t}\right|^{2}$ are uniformly bounded, we may apply the functional calculus to conclude that

$$
\left|D_{t}\right| \rightarrow|H-K| \text { strongly . }
$$


Finally one has for some $M$ and any orthonormal basis $\left\{\psi_{i}\right\}$

$$
\begin{aligned}
M & \geqq \varlimsup_{t \rightarrow 0}\left\|\left(\mathrm{U}_{t}-\mathrm{V}_{t}\right) / \mathrm{t}\right\|_{\mathrm{Tr}} \\
& =\varlimsup_{t \rightarrow 0} \sum_{i \geqq 1}\left(\left|\mathrm{D}_{t}\right| \psi_{i} \mid \psi_{i}\right) \\
& \geqq \sum_{i=1}^{N} \lim _{t \rightarrow 0}\left(\left|D_{t}\right| \psi_{i} \mid \psi_{i}\right) \\
& =\sum_{i=1}^{N}\left(|H-K| \psi_{i} \mid \psi_{i}\right) \text { for any integer } N .
\end{aligned}
$$

Then $\|H-K\|_{\mathrm{Tr}}<\infty$.

3. $\Rightarrow 4$.

We again have the integral relation

$$
\left(\frac{\alpha_{t}-\beta_{t}}{t}\right)(A)=\frac{1}{t} \int_{0}^{t} d s \alpha_{s}\left(\delta_{H}-\delta_{K}\right) \beta_{t-s}(A)
$$

and so $\left\|\frac{\alpha_{t}-\beta_{t}}{t}\right\| \leqq\left\|\delta_{H}-\delta_{K}\right\|$

$4 . \Rightarrow 3$.

Applying the remarks preceding this theorem we may lift the automorphism groups $\alpha_{t}, \beta_{t}$ to automorphism groups of the von Neumann algebra $\mathscr{M}$ associated with the Fock space representation [in this case $\left.\mathscr{M}=\mathscr{L}\left(\mathscr{H}_{F}\right)\right]$. We write $\tilde{\alpha}_{t}, \tilde{\beta}_{t}$ for the extensions. These are adjoint semi-groups and the operator norm of $\left(\tilde{\alpha}_{t}-\tilde{\beta}_{t}\right) / t$ in $\mathscr{M}$ is by Kaplansky's density theorem, [15], equal to that of $\left(\alpha_{t}-\beta_{t}\right) / t$ on $\mathfrak{U}$.

Let $\tilde{\delta}_{H}$ and $\tilde{\delta}_{K}$ be the generators of the extended automorphism groups. Then Theorem 2 of [14] shows that $\mathscr{D}\left(\tilde{\delta}_{H}\right)=\mathscr{D}\left(\tilde{\delta}_{K}\right)$ and $\left\|\tilde{\delta}_{H}-\tilde{\delta}_{K}\right\|<\infty$ on this common domain. We claim that the proof will be finished once we show that $\mathscr{D}\left(\delta_{H}\right) \cap \mathscr{D}\left(\delta_{K}\right)$ is a joint core of $\delta_{H}$ and $\delta_{K}$. Indeed we know that $\tilde{\delta}_{H}-\tilde{\delta}_{K}$ is a bounded derivation, say $\delta$, on the joint strong continuity subspace of $\tilde{\alpha}$ and $\tilde{\beta}$. Then $\delta\left(\mathscr{D}\left(\delta_{H}\right) \cap \mathscr{D}\left(\delta_{K}\right)\right) \cong \mathfrak{U}$ and so by closure $\delta(\mathfrak{U}) \subseteq \mathfrak{U}$; that is $\delta$ extends to a bounded derivation of $\mathfrak{A}$. Since $\delta_{H}=\delta_{K}+\delta$ on $\mathscr{D}\left(\delta_{H}\right) \cap \mathscr{D}\left(\delta_{K}\right)$, it then follows by taking the closure on both sides that $\mathscr{D}\left(\delta_{H}\right)=\mathscr{D}\left(\delta_{K}\right)$ and $\left\|\delta_{H}-\delta_{K}\right\|<\infty$.

We now complete the argument by showing that $\mathscr{D}\left(\delta_{H}\right) \cap \mathscr{D}\left(\delta_{K}\right)$ is indeed a joint core of $\delta_{H}$ and $\delta_{K}$.

By restricting $\alpha_{t}, \beta_{t}$ to any $a(f)$ one has, using $\|a(f)\|=\|f\|$, that $\left\|\left(U_{t}-V_{t}\right) / t\right\| \leqq M$. Here the norm is the usual operator norm on $\mathscr{H}$. One then has, using $[14$, Corollary 3$]$, that $\mathscr{D}(H)=\mathscr{D}(K)$. Taking $f$ 's from the latter subspace and generating the corresponding monomials in the $a(f)$ 's gives a subspace of $\mathscr{D}\left(\delta_{H}\right) \cap \mathscr{D}\left(\delta_{K}\right)$ which is dense in $\mathscr{U}$. Since $e^{i t H} \mathscr{D}(H)=\mathscr{D}(H), e^{i t H} \mathscr{D}(K)=\mathscr{D}(K)$, the 
subspace is furthermore invariant under the action of $\alpha_{t}$ and $\beta_{t}$. Hence it is a core for both $\delta_{H}$ and $\delta_{K}$ by [21], Theorem 3 .

1. $\Leftrightarrow 3$.

This equivalence is essentially a restatenent of Araki's result [2] since the derivation induced by $H-K$ coincides with $\delta_{H}-\delta_{K}$.

We now give an abstract version of part of the previous theorem. It is necessary to impose an additional restriction as we show in Examples 2.5 and 4.6. The Fock space argument of the previous theorem can be replaced by a more general argument.

Theorem 4.4. Let $\mathfrak{A}$ be a simple $C^{*}$-algebra with identity and strongly continuous

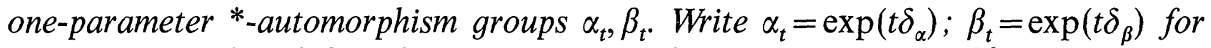
derivations $\delta_{\alpha}, \delta_{\beta}$ of $\mathfrak{U}$. If $\left\|\alpha_{t}-\beta_{t}\right\|=O(t)$ then there exists a (faithful) representation $\pi$ of $\mathfrak{U}$ such that $\alpha_{t}, \beta_{t}$ extend to $\sigma$-weakly continuous $*_{\text {-automorphism }}$ groups $\tilde{\alpha}_{t}, \tilde{\beta}_{t}$ of $\pi(\mathfrak{Q})^{\prime \prime}$. If $\delta_{\tilde{\alpha}}, \delta_{\tilde{\beta}}$ denote the corresponding generators of $\tilde{\alpha}_{t}, \tilde{\beta}_{t}$ then $\mathscr{D}\left(\delta_{\tilde{\alpha}}\right)=\mathscr{D}\left(\delta_{\tilde{\beta}}\right)$ and $\left\|\delta_{\tilde{\alpha}}-\delta_{\tilde{\beta}}\right\|<\infty$. If $\mathscr{D}\left(\delta_{\alpha}\right) \cap \mathscr{D}\left(\delta_{\beta}\right)$ is in addition a joint core of $\delta_{\alpha}$ and $\delta_{\beta}$ then $\left\|\alpha_{t}-\beta_{t}\right\|=O(t)$ is equivalent with $\mathscr{D}\left(\delta_{\alpha}\right)=\mathscr{D}\left(\delta_{\beta}\right)$ and $\left\|\delta_{\alpha}-\delta_{\beta}\right\|<\infty$.

Proof. Consider the orbit of any state under the action of $t \rightarrow \alpha_{t}$. By applying an invariant mean we obtain a state $\omega$ which is invariant for the automorphism group $\alpha_{t}$ i.e. $\omega\left(\alpha_{t}(x)\right)=\omega(x)$ for all $t \in \boldsymbol{R}$.

Via the GNS procedure, one has a Hilbert space $\mathscr{H}_{\omega}$, a representation $\pi_{\omega}$ of $\mathfrak{A}$; and a unitary representation of $\boldsymbol{R}, t \rightarrow V_{t} \in \mathscr{L}\left(\mathscr{H}_{\omega}\right)$, such that

$$
\pi_{\omega}\left(\alpha_{t}(x)\right)=V_{t} \pi_{\omega}(x) V_{-t}=\tilde{\alpha}_{t}\left(\pi_{\omega}(x)\right)
$$

The representation is faithful since $\mathfrak{U}$ is simple. Next note that for $t$ sufficiently small

$$
\left\|\alpha_{t} \cdot \beta_{-t}-\imath\right\|<2
$$

Here $l$ is the identity automorphism. Results of Kadison and Ringrose [11], then give a unitary $U_{t} \in \mathscr{M}=\pi_{\omega}(\mathfrak{U})^{\prime \prime}$ such that

$$
\pi_{\omega}\left(\alpha_{t}\left(\beta_{-t}(x)\right)\right)=V_{t} \pi_{\omega}\left(\beta_{-t}(x)\right) V_{-t}=U_{t} \pi_{\omega}(x) U_{t}^{*} .
$$

Unwinding one has that

$$
\pi_{\omega}\left(\beta_{-t}(x)\right)=V_{-t} U_{t} \pi_{\omega}(x) U_{t}^{*} V_{t}, \text { for small } t .
$$

Thus $\beta_{t}$ extends to an automorphism $\tilde{\beta}_{t}$ of $\mathscr{M}$ for all sufficiently small $t$, and by iteration, for all $t$. The extension is moreover $\sigma$-weakly continuous in $t$, for given $x \in \mathscr{M}$ and $\phi \in \mathscr{M}_{*}$ one has

$$
\left|\phi\left(\beta_{t}(x)-x\right)\right| \leqq\left|\phi\left(\alpha_{t}(x)-x\right)\right|+\left\|\alpha_{t}(x)-\beta_{t}(x)\right\|
$$


But by Kaplansky's density theorem [15],

$$
\begin{aligned}
\sup _{x \in \mathcal{M}}\left\|\frac{\alpha_{t}(x)-\beta_{t}(x)}{\|x\|}\right\| & =\sup _{x \in \mathfrak{A}}\left\|\frac{\alpha_{t}(x)-\beta_{t}(x)}{\|x\|}\right\| \\
& \leqq M|t|
\end{aligned}
$$

for $t$ small. Thus continuity follows by the group property.

We now have dual semi-groups and so may conclude the first part of the theorem by applying [14, Theorem 2].

If in addition one has that $\mathscr{D}\left(\delta_{\alpha}\right) \cap \mathscr{D}\left(\delta_{\beta}\right)$ is a joint core of $\delta_{\alpha}$ and $\delta_{\beta}$ we reach our conclusion by arguing as in 4$) \Rightarrow 3$ ) of the previous theorem.

Conversely suppose that $\mathscr{D}\left(\delta_{\alpha}\right)=\mathscr{D}\left(\delta_{\beta}\right)$ and $\left\|\delta_{\alpha}-\delta_{\beta}\right\|<\infty$. Then choosing $x$ in the common domain one has the integral relation.

$$
\alpha_{t}(x)-\beta_{t}(x)=\int_{0}^{t} d s \alpha_{s}\left(\delta_{\alpha}-\delta_{\beta}\right) \beta_{t-s}(x) .
$$

The $O(t)$ behaviour is then clear since such $x$ are dense. The general setting for the first two examples will be a $C^{*}$-algebra, with unit acting on a Hilbert space $\mathscr{H}$ and two strongly continuous one parameter automorphism groups $\alpha_{t}, \beta_{t}$ of $\mathfrak{A}$ which extend to weakly continuous automorphism groups of $\mathfrak{A}^{\prime \prime}$. Let $\delta_{\alpha}, \delta_{\beta}$ be the generators of $\alpha_{t}, \beta_{t}$ respectively as automorphism groups of $\mathfrak{A}$, and $\delta_{\alpha}, \hat{\delta}_{\beta}$ the corresponding generators as groups of automorphisms of $\mathfrak{U}^{\prime \prime}$. It is clear that $\delta_{\alpha} \subseteq \hat{\delta}_{\alpha}, \delta_{\beta} \subseteq \hat{\delta}_{\beta}$. Moreover $\mathscr{D}\left(\delta_{\alpha}\right)=\left(1+\lambda \hat{\delta}_{\alpha}\right)^{-1}(\mathfrak{U}), \mathscr{D}\left(\delta_{\beta}\right)=\left(1+\lambda \hat{\delta}_{\beta}\right)^{-1}(\mathfrak{U})$ for any real $\lambda$.

In the following two examples $\delta_{\alpha}$ is obtained from $\delta_{\beta}$ in the following manner : We choose a unitary $v \in M$ such that $v \in \mathscr{D}\left(\hat{\delta}_{\alpha}\right), v \notin \mathscr{D}\left(\delta_{\alpha}\right)$ and set

$$
\delta_{\beta}(x)=v^{*} \delta_{\alpha}\left(v x v^{*}\right) v=\sigma^{-1}\left(\delta_{\alpha}(\sigma(x))\right),
$$

where $\sigma(x)=v x v^{*}$ is an inner automorphism. This relation persists for the extensions $\hat{\delta}_{\alpha}, \hat{\delta}_{\beta}$ so that one has

a) $\mathscr{D}\left(\delta_{\beta}\right)=v^{*} \mathscr{D}\left(\delta_{\alpha}\right) v$;

b) $\mathscr{D}\left(\hat{\delta}_{\beta}\right)=\mathscr{D}\left(\hat{\delta}_{\alpha}\right)$;

c) $\hat{\delta}_{\beta}(x)=v^{*} \hat{\delta}_{\alpha}(x) x v^{*} v+v^{*} v \hat{\delta}_{\alpha}(x) v^{*} v$

$$
\begin{aligned}
& +v^{*} v x \hat{\delta}_{\alpha}\left(v^{*}\right) v \\
= & \hat{\delta}_{\alpha}(x)+\left[v^{*} \hat{\delta}_{\alpha}(v), x\right] .
\end{aligned}
$$

The last two conditions show that $\left\|\alpha_{t}-\beta_{t}\right\|=O(t), t \rightarrow 0$, as may be seen by applying Theorem 2 of [15] or computing directly. We shall show that $v$ may be chosen so as to make

$$
\mathscr{D}\left(\delta_{\beta}\right) \cap \mathscr{D}\left(\delta_{\alpha}\right)=v^{*} \mathscr{D}\left(\delta_{\alpha}\right) v \cap \mathscr{D}\left(\delta_{\alpha}\right)
$$

a non-dense subspace of $\mathfrak{A}$.

Example 4.5. In this example $\mathfrak{A}$ is the common strong continuity subspace of $\alpha$ and $\beta$ but $\mathscr{D}\left(\delta_{\alpha}\right) \cap \mathscr{D}\left(\delta_{\beta}\right)$ in not even $\sigma$-weakly dense in $\mathfrak{U}$.

Let $M_{2}$ be the complex $2 \times 2$ matrices and $C(\boldsymbol{T})$ the (complex valued) continuous functions on the circle group $\boldsymbol{T}$ with the usual supremum norm. For $\mathfrak{A}$ 
we take $M_{2} \otimes C(T)$ and consider $\mathfrak{A}$ represented on $=\not^{2} \otimes L^{2}(T)=L^{2}(T) \oplus L^{2}(T)$, using Haar measure on $\boldsymbol{T}$.

Then $\mathfrak{I}^{\prime \prime}=M_{2} \otimes L^{\infty}(T)$, i.e. the von Neumann algebra of all $2 \times 2$ matrices with entries in $L^{\infty}(T)$.

Define $\alpha_{t}=l \otimes \tau_{t}$, where $l$ is the identity automorphism and $\left(\tau_{t} f\right)(x)=f(x-t)$ in the group defined by rotation of the circle (translations $\bmod 2 \pi$ ). Then $\alpha_{t}$ is $\sigma$-weakly continuous on $\mathfrak{Q}^{\prime \prime}$ and $\mathfrak{A}$ is just the strong continuity subspace of $\alpha_{t}$.

Next let $\theta: \boldsymbol{T} \rightarrow \boldsymbol{R}$ be a uniformly Hölder continuous function i.e. $\left|\theta\left(t_{2}\right)-\theta\left(t_{1}\right)\right|$ $<M\left|t_{2}-t_{1}\right|$ for some constant $M$, but $\theta$ is non-differentiable on a dense set of points of. One such function $\theta$ is

$$
\theta(s)=\sum_{n-1}^{\infty} \frac{1}{n !}\left|s-r_{n}\right|,
$$

where $r_{n}$ is an enumeration of the rationals in $T$. Let $\delta_{\tau}$ and $\hat{\delta}_{\tau}$ be the generators of $\tau_{t}$ viewed as an automorphism group of $C(\boldsymbol{T}), L^{\infty}(\boldsymbol{T})$ respectively. Then $\theta \in \mathscr{D}\left(\hat{\delta}_{\tau}\right)$ (it is absolutely continuous) and $\theta \notin \mathscr{D}\left(\delta_{\tau}\right)$. Further $\left\|\hat{\delta}_{\tau}(\theta)\right\| \leqq M$. We verify directly that $e^{i \theta}$ is also Hölder continuous and hence in $\mathscr{D}\left(\hat{\delta}_{\tau}\right)$. Clearly $e^{i \theta} \notin \mathscr{D}\left(\delta_{\tau}\right)$.

Next define the unitary operator $v$ by

$$
\boldsymbol{T} \ni S \rightarrow\left(\begin{array}{cc}
1 & 0 \\
0 & e^{i \theta(s)}
\end{array}\right) .
$$

Then $v \in \mathscr{D}\left(\hat{\delta}_{\alpha}\right), v \notin \mathscr{D}\left(\delta_{\alpha}\right)$ and $v$ is a unitary in $\mathfrak{U}$.

Given

$$
\left(\begin{array}{ll}
f_{11} & f_{12} \\
f_{21} & f_{22}
\end{array}\right) \in \mathfrak{A},
$$

we have

$$
v^{*}\left(\begin{array}{ll}
f_{11} & f_{12} \\
f_{21} & f_{22}
\end{array}\right) v=\left(\begin{array}{cc}
f_{11} & e^{i \theta} f_{12} \\
e^{-i \theta} f_{21} & f_{22}
\end{array}\right) .
$$

Now $\mathscr{D}\left(\delta_{\alpha}\right)$ consists of just the matrices $\left(f_{i j}\right)$ where each $f_{i j}$ is continuously differentiable: Since these functions are continuous $f$ and $e^{i \theta} f$ can both be continuously differentiable only when $f=0$. This shows that

$$
\mathscr{D}\left(\delta_{\alpha}\right) \cap \mathscr{D}\left(\delta_{\beta}\right)=\left\{\left(\begin{array}{cc}
f_{11} & 0 \\
0 & f_{22}
\end{array}\right) \mid f_{i i} \in \mathscr{D}\left(\delta_{\tau}\right)\right\}
$$

so that $\mathscr{D}\left(\delta_{\alpha}\right) \cap \mathscr{D}\left(\delta_{\beta}\right)$ is not dense in $\mathfrak{A}$.

Example 4.6. This example is a variation of the first and provides us with a simple $C^{*}$-algebra with unit where $\mathscr{D}\left(\delta_{\alpha}\right) \cap \mathscr{D}\left(\delta_{\beta}\right)$ is not dense in $\mathfrak{A}$ and $\left\|\alpha_{t}-\beta_{t}\right\|=O(t)$.

We refer to $\mathfrak{A}, \alpha$, and $v$ of the previous example as $\mathfrak{A}^{0}, \alpha^{0}, v^{0}$. Let $t_{0}$ be an irrational rotation of the circle and define

$$
\tau=\alpha_{t_{0}}^{0} \text {. }
$$


Then $\tau$ is an automorphism of $\mathfrak{A}^{0}$, which acts freely and ergodically on its spectrum. It follows from $[9,16]$ that the $C^{*}$-crossed product $\mathfrak{A}=C^{*}\left(\mathfrak{A}^{0}, \tau\right)$ $=M_{2} \otimes C^{*}(C(T), \tau)$ is simple. Since this crossed product is discrete we have a canonical imbedding of $\mathfrak{A}^{0}$ into $\mathfrak{A}$ and since $\alpha_{t}^{0}$ commutes with $\tau, \alpha_{t}^{0}$ extends canonically to a strongly continuous group $\alpha_{t}$ of automorphisms of $\mathfrak{A}$. Using Zeller-Meier's approach [19] we define the von Neumann crossed product of $\mathfrak{A}^{0 \prime}=M_{2} \otimes L^{\infty}(\boldsymbol{T})$ by $\tau$ as $V N\left(\mathfrak{A}^{0 \prime \prime}, \tau\right)$. The simplicity of $\mathfrak{A}$ allows us to represent $\mathfrak{A}$ faithfully in $V N\left(\mathfrak{A}^{0 \prime \prime}, \tau\right)$. Keeping the same letter we see that $\mathfrak{I}^{\prime \prime}=V N\left(\mathfrak{Q}^{0 \prime \prime}, \tau\right)$ and $\alpha_{t}$ extends to a $\sigma$-weakly continuous group of automorphisms of $V N\left(\mathfrak{H}^{0 \prime \prime}, \tau\right)$. Now $v=v^{0} \in \mathfrak{A}^{0} \cong \mathfrak{A}$ and so $v \in \mathscr{D}\left(\hat{\delta}_{\alpha}\right), v \notin \mathscr{D}\left(\delta_{\alpha}\right)$. One can then show, as in Example 1, that

$$
\mathscr{D}\left(\delta_{\alpha}\right) \cap \mathscr{D}\left(\delta_{\beta}\right) \cong C^{*}\left(\mathfrak{H}^{00}, \tau\right) \text {, }
$$

viewed as a subalgebra of $V N\left(\mathfrak{H}^{0 \prime \prime}, \tau\right)$, where $\left.\mathfrak{A}^{00}=\left\{\left(\begin{array}{cc}f_{11} & 0 \\ 0 & f_{22}\end{array}\right)\right) f_{i j} \in \mathscr{D}\left(\delta_{\tau}\right)\right\}$.

The techniques used in the first two examples can be used to show that the saturation property of de Leeuw [13] does not serve to characterize the domain of the generator of a one parameter automorphism group of a simple $C^{*}$-algebra with unit. This property says that given a one parameter group of automorphism $\tau_{t}$ the domain of its generator is identical with those $x$ such that $\left\|\tau_{t}(x)-x\right\|=O(t)$.

Example 4.7. There exists a simple $C^{*}$-algebra $\mathfrak{A}$ with unit, a one parameter group. $\tau_{t}$, of automorphisms of $\mathfrak{A}$ with generator $\delta$ and an element $x \in \mathfrak{A}$ such that $\left\|\tau_{t}(x)-x\right\|=O(t), t \rightarrow 0$, but $x \notin \mathscr{D}(\delta)$. The simple $C^{*}$-algebra $\mathfrak{A}$ is the $C^{*}$-crossed product of $\mathfrak{A}_{0}=C(\boldsymbol{T})$ by an irrational rotation. The group of automorphisms $\tau_{t}$ is that gotten by lifting to the crossed product the automorphism of $C(T)$ given by rotation through an angle $t$. Call this automorphism $\tau_{t}^{0}$. Since $\mathfrak{U}_{0}$ is embedded in $\mathfrak{A}$ we have $\delta_{0} \leqq \delta$ and $\mathscr{D}\left(\delta_{0}\right)=\mathscr{D}(\delta) \cap \mathfrak{A}_{0}$ where $\delta_{0}, \delta$ are the generators of $\tau_{t}^{0}, \tau_{t}$ respectively. If we take for $x \in \mathfrak{A}_{0}$ a Hölder continuous function $f$, which is nondifferentiable, one has that $x \notin \mathscr{D}\left(\delta_{0}\right)$ and by the above remark $x \notin \mathscr{D}(\delta)$.

We show in the next example that commutativity is required for Proposition 4.1. The following two examples delineate the distinction between our results and those obtained in [5].

Example 4.8. For any $0 \leqq \varepsilon \leqq 2$ there exists two strongly continuous oneparameter unitary groups $U_{t}, V_{t}$ on a separable Hilbert space $\mathscr{H}$ such that

$$
\left\|U_{t}-V_{t}\right\|=\varepsilon \quad t \in \boldsymbol{R} \backslash\{0\} .
$$

Proof. Let $\mathscr{H}=L^{2}(R)$ and define $U_{t}$ by

$$
\left(U_{t} f\right)(s)=f(s-t) .
$$

For $\varepsilon$ given, choose $\delta=\arcsin (\varepsilon / 2)$ and define a function $\theta$ by

$$
\theta(s)=\delta \sin \left(s^{2}\right) .
$$

Next define a unitary operator $W$ on by

$$
(W f)(s)=e^{i \theta(s)} f(s)
$$


and define

$$
V_{t}=W U_{t} W^{*}
$$

Then

$$
\left\|V_{t}-U_{t}\right\|=\left\|W U_{t} W^{*} U_{-t}-1\right\| .
$$

By an easy calculation:

$$
\left(W U_{t} W^{*} U_{-t} f\right)(s)=e^{i(\theta(s)-\theta(s-t))} f(s)
$$

so

$$
\left\|V_{t}-U_{t}\right\|=\sup _{s}\left|e^{i(\theta(s)-\theta(s-t))}-1\right| .
$$

It is then clear that

$$
\left\|U_{t}-V_{t}\right\| \leqq\left|e^{i(\delta+\delta)}-1\right|=\varepsilon
$$

for all $t$. Next fix $t \neq 0$ and $\varepsilon^{\prime}>0$. We will exhibit an $s$ such that

$$
\begin{aligned}
& \theta(s)=\delta \\
& |\theta(s-t)+\delta|<\varepsilon^{\prime}
\end{aligned}
$$

i.e.

$$
|\theta(s)-\theta(s-t)-2 \delta|<\varepsilon^{\prime}
$$

This will end the proof. It amounts to showing that there exists integers $n, m$ such that simultaneously:

$$
\begin{aligned}
& s^{2}-(s-t)^{2} \cong \pi+2 n \pi \\
& s^{2}=\frac{\pi}{2}+2 m \pi
\end{aligned}
$$

Since $s^{2}-(s-t)^{2}=2 s t-t^{2}$, this amounts to finding $n, m$ such that

$$
2 \sqrt{\frac{\pi}{2}+2 m} t-t^{2} \cong \pi+2 n \pi \text {. }
$$

But since the derivative of the function $x \rightarrow \sqrt{x}$ tends to 0 as $x \rightarrow+\infty$, while the function itself tends to $\infty$, this is clearly possible for any $\varepsilon^{\prime}$. This shows that $\left\|U_{t}-V_{t}\right\| \geqq \varepsilon$ for $t \neq 0$ i.e.

$$
\left\|U_{t}-V_{t}\right\|=\varepsilon \quad t \neq 0 .
$$

One easily refines the technique of the proof given here to show that $\operatorname{Sp}\left(W U_{t} W^{*} U_{-t}\right)=e^{i[-2 \delta, 2 \delta]}$. This implies the following:

Example 4.9. If $\mathscr{M}=\mathscr{L}(\mathscr{H}), \mathscr{H}$ a separable Hilbert space, then for any $\delta$ such that $0 \leqq \delta \leqq 2$ there exists two $\sigma$-weakly continuous one-parameter groups $\alpha_{t}, \beta_{t}$ of *-automorphisms of $\mathscr{L}(\mathscr{H})$ such that

$$
\left\|\alpha_{t}-\beta_{t}\right\|=\delta \quad t \in \boldsymbol{R} \backslash\{0\} .
$$


Proof. Use $U_{t}, V_{t}$ from Example 4.8 with $\varepsilon=\sqrt{2\left(1-\sqrt{1-\varepsilon^{2}} / 4\right)}$ and set $\alpha_{t}=U_{t} \circ U_{t}^{*}, \beta_{t}=V_{t} \circ V_{t}^{*}$. Then

$$
\begin{aligned}
\left\|\beta_{t}-\alpha_{t}\right\| & =\left\|\beta_{t} \alpha_{-t}-i\right\| \\
& =\left\|\operatorname{ad}\left(W U_{t} W^{*} U_{-t}\right)-\operatorname{ad}(1)\right\|=\delta .
\end{aligned}
$$

Next we give a physically more interesting example of unitary groups such that $\left\|U_{t}-V_{t}\right\|<\varepsilon$ for all $t$.

Example 4.10 (Smooth Interactions). Let $U_{t}, V_{t}$ be two unitary groups on the Hilbert space $\mathscr{H}$ with generators $H_{0}$ and $H=H_{0}+V$ where $V$ is a self-adjoint operator such that

$$
\left\||V|^{1 / 2}\left(H_{0}-z\right)^{-1}|V|^{1 / 2}\right\| \leqq N<1
$$

for all $z$ with $\operatorname{Im} z \neq 0$. It follows by a perturbation calculation that

$$
\left\||V|^{1 / 2}(H-z)^{-1}|V|^{1 / 2}\right\| \leqq N(1-N)^{-1}, \quad \operatorname{Im} z \neq 0 .
$$

Moreover if $\|A\|_{H}$ is defined by

$$
\left\|A_{H}\right\|^{2}=\sup _{\psi \in \mathscr{H}} \int_{-\infty}^{\infty} d t\left\|A e^{i H t} \psi\right\|^{2} /\|\psi\|^{2}
$$

then one may deduce [30] that

$$
\left\||V|^{1 / 2}\right\|_{H_{0}}^{2}<2 N, \quad\left\||V|^{1 / 2}\right\|_{H}^{2}<2 N(1-N)^{-1} .
$$

But one then has

$$
\begin{aligned}
\left|\left(\varphi,\left(U_{t}-V_{t}\right) \psi\right)\right| & =\left|\int_{0}^{t} d s\left(\varphi, U_{s} V V_{t-s} \psi\right)\right| \\
& \leqq\|\varphi\|\|\psi\|\left\||V|^{1 / 2}\right\|_{H_{0}}\left\||V|^{1 / 2}\right\|_{H}
\end{aligned}
$$

Therefore

$$
\left\|U_{t}-V_{t}\right\| \leqq 2 N(1-N)^{1 / 2} .
$$

[In particular if $N<(\sqrt{5}-1) / 2$ then $\left\|U_{t}-V_{t}\right\|<2$ for all $t \in \boldsymbol{R}$.] It is also possible to show that

$$
\left\|\left(H_{0}-z\right)^{1 / 2}\left(U_{t}-V_{t}\right)(H-z)^{1 / 2}\right\| \leqq 2|t| N(1-N)^{1 / 2} .
$$

A specific situation in which this example applies is given by $=L^{2}\left(\boldsymbol{R}^{3}\right)$, $H_{0}=-\nabla^{2}$ and $V$ a multiplication operator $(V \psi)(x)=V(x) \psi(x)$ such that $V(x)$ is real and

$$
(1 / 4 \pi)\left[\int d x d y|V(x)||V(y)| /(x-y)^{2}\right]^{1 / 2}<1 .
$$

This example occurs in scattering theory and one can show that

$$
W=\underset{t \rightarrow \infty}{\operatorname{strong} \operatorname{limit}} U_{t} V_{-t}
$$

exists, is unitary, and $U_{t} W=W V_{t}$ (see for example, [31]). 
Acknowledgement. We are pleased to acknowledge useful conversations with H. Araki. G. Elliot, U. Haagerup, R. V. Kadison, C. Moore, J. Roberts, and M. Takesaki. For invitations which made this collaboration possible we are all grateful to Ludwig Streit at the Universität Bielefeld, the first two authors to the Universite de Toulon and C.N.R.S. Marseille, the first and third named authors to the Mathematics Department at The Pennsylvania State University. The first named author is grateful for support from the Norwegian Research Council for Science and Humanities and the second named for the support of the Alexander von Humboldt Stiftung.

\section{References}

1. Araki,H. : Some properties of modular conjugation operator of von Neumann algebras and a noncommutative Radon-Nikodym theorem with a chain rule. Pacific J. Math. 50, 309-354 (1974)

2. Araki,H.: On quasi-free states of CAR and Bogoliubov automorphism. Publ. RIMS 6, 385-442 $(1971 / 71)$

3. Araki,H. : Relative Hamiltonian for faithful normal states of a von Neumann algebra. Publ. RIMS 9, 165-209 )1973)

4. Bratteli,O., Robinson,D.W.: Unbounded derivations of von Neumann algebras. Ann. Inst. Henri Poincaré

5. Buchholz,D., Roberts,J.E.: Bounded perturbations of dynamics. Commun. math. Phys. 49, 161177 (1976)

6. Butzer,P.L., Berens,H.: Semi-groups of operators and approximation. Berlin-Heidelberg-New York: Springer 1967

7. Connes, A.: Characterization des espaces vectoriels ordonnés sous-jacents aux algèbres de von Neumann. Ann. Inst. Four. 24, 121-155 (1974)

8. Connes, A. : Etats presque periodiques sur une algebra de von Neumann C.R. Acad Sc. (Paris) 274, $1402-1405(1972)$

9. Effros, E., Hahn,F.: Locally compact transformation groups and $C^{*}$-algebras. Mem. Am. Math. Soc. 75 (1967)

10. Haagerup, U.: The standard form of von Neumann algebras. Math. Scand. 271-283 (1976)

11. Kadison, R.V., Ringrose,J.R.: Derivations and automorphisms of operator algebras. Commun. math. Phys. 4, 32-63 (1967)

12. Leeuw, K.de: On the adjoint semigroup and some problems in the theory of approximation. Math. Z. 73, 219-234 (1960)

13. Powers, R.T.: UHF algebras and their application to representation of the anticommutation relations. In: Cangère lectures in physics, Vol. 4 (ed. D. Kastler), pp. 137-168 (1967)

14. Robinson, D. W.: The approximation of flows. J. func. Anal. 24, 280-290 (1977)

15. Sakai, S.: $C^{*}$-algebras and $W^{*}$-algebras. Berlin-Heidelberg-New York: Springer 1974

16. Takai,H.: The quasi-orbit space of continuous $C^{*}$-dynamical systems. Trans. Am. math. Soc. 216, $105-113(1976)$

17. Takesaki,M.: Tomitas theory of modular Hilbert algebras and its applications. Lecture notes in mathematics, Vol. 128. Berlin-Heidelberg-New York: Springer 1970

18. Yosida,K. : Functional analysis, 4ed. Berlin-Heidelberg-New York: Springer 1974

19. Zeller-Meier,G. : Produits croisés d'une $C^{*}$-algèbre par une groupe d'automorphismes. J. Math. Pure Appl. 47, 101-239 (1968)

20. Longo, R.: On perturbed derivations of $C^{*}$-algebras. Roma, preprint

21. Bratteli,O., Robinson,D.W.: Unbounded derivations of $C^{*}$-algebras. II. Commun. math. Phys. 46, $11-30$ (1976)

22. Kadison, R. : Derivations of operator algebras. Ann. Math. 83, 280-293 (1966)

23. Arveson, W.: On groups of automorphisms of operator algebras. J. func. Anal. 15, $217-243$ (1974)

24. Connes, A. : Une classification des facteurs de type III. Ann. Sci. Ec. Norm. Sup. 6, $133-252$ (1973)

25. Olesen,D.: On norm-continuity and compactness of spectrum. Math. Scand. 35, $223-236$ (1974)

26. Reynolds, M.: Perturbations of groups of automorphisms of von Neumann algebras. Proc. Am. Math. Soc. 55, 326-328 (1976)

27. Arveson,W.: An inviation to $C^{*}$-algebras. Berlin-Heidelberg-New York: Springer 1976

28. Hansen, F., Olesen, D. : Perturbations of centre-fixing dynamical system. University of Copenhagen, Preprint (1976) 
29. Kadison, R.V.: Transformations of states in operator theory and dynamics. Topology 3, 177-198 (1965)

30. Kato, T.: Wave operators and similarity for non self-adjoint operators. Math. Ann. 163, 258-279 (1966)

31. Simon, B.: Quantum mechanics for Hamiltonian defined as quadratic forms. Princeton: University Press 1971

32. Bonsall, Duncan: Numerical ranges of operators on normed spaces and elements of normed algebras. Cambridge: University Press

33. Woronowicz,S.L.: A remark on the polar decomposition of $m$-sectorial operators. ZIF, Preprint

34. Fujii, M., Furuta,T., Matsumoto,K.: Equivalence of operator representations of semi-groups. Math. Japonicae 20, 253-256 (1975)

Communicated by J. Glimm

Received September 27, 1977; in revised form December 5, 1977 